\title{
An Ecological Model for Sustainable Forest Management of Eco-system Based on Optimal Control Theory
}

\author{
Md. Nazmul Hasan ${ }^{1}$, Md. Haider Ali Biswas ${ }^{2}$, Md. Sharif Uddin ${ }^{3}$ \\ ${ }^{1}$ Department of Mathematics, Jahangirnagar University, Dhaka, Bangladesh. \\ 2 Mathematics Discipline, Khulna University, Bangladesh. \\ ${ }^{3}$ Department of Mathematics, Jahangirnagar University, Dhaka, Bangladesh. \\ Correspondence to: Md. Nazmul Hasan, Email: nazmulmaths@gmail.com
}

\begin{abstract}
Sustainable forest management is one of the challenging issues in the present century. In this manuscript, we have employed the model of control theory to control the consequence of toxicity and illegal logging of mature trees in the ecosystem of Sundarban, the largest mangrove forest in the world. In this investigation, we have momentarily mentioned some of the fields in which these challenges are present. These fields especially consist of sustainable forest management of ecosystem. We have reflected on the modified Leslie-Gower response function to set up as the alternative resource for industries when forestry resources are devastated. The boundedness, persistence, equilibria and stability are examined along with bionomic equilibria and optimal harvesting strategy. Our main aim is to investigate the spans and applications of control theory in real life situation, especially in efficient and sustainable forest growth.
\end{abstract}

Keywords: Toxicity, Persistence, Lyapunov function, Optimal harvesting.

\section{Introduction}

The Sundarban is the single largest mangrove forest in the world comprising a total area of 9827 sq. kms. lying in both Bangladesh and India. It is the only marshy mangrove land included in the list of world heritage sites. This area shows high biodiversity with unique flora and fauna. Some of the biological species have already been driven to extinction. Trees and many others are at the verge of extinction due to several external forces such as over exploitation over harvesting, environmental pollution and mismanagement of forestry. Environmental pollution, natural catastrophes and mismanagement of the habitat may cause reduction of species population. Hazards such as coastal flooding and cyclone also cause the decline of species in an ecosystem.

A number of species have become extinct from the Sundarban during the last 100 years. It provides insitu conservation of biodiversity of natural and semi-natural ecosystems and landscapes and contributes to sustainable economic development of the human population living within and around the biosphere reserve.

Both biotic and abiotic are dependent component to each other and their balance is essential for the sustainability and stability of the world. Forests are necessary for life on earth, for mitigating and adapting to climate change, ensuring adequate supply of fresh water, enhancing biodiversity and providing sustainable income and livelihood, including food safety. But it is now under unprecedented and unrelenting pressure. The United Nation has declared 'the year 2011' was the International Year of "Forests" by to upraise awareness and strengthen the sustainable management, conservation and sustainable development of all types of forests. In [24] the author reviews both the effect of deforestation on climate change and the impact of climate change on forests. At present forests cover about $31 \%$ of world's area [23]. During the last five decades different measure are taken for the conservation of fishery applying different harvesting and optimal control strategies. The first research on fishery management based on optimal control is conducted by Clark in the year 1976 [25], which are followed by K. Chaudhuri [5]. Like fishery, forestry, conservation is also a key factor responsible for the stability of the ecosystem. I have paid attention to optimal control for the proper management of forestry resources, mainly tree biomass. The concept of modeling through rates of change is well known in physics and engineering. The idea behind the generalization is to obtain the system through a suitable number of differential equations with considered state variable. Outputs are 
presented as the current state functions.

The forestry biomass and forestry resource based industrialization is similar to the predator-prey dynamical system. The formulation of Leslie-Gower [14] predator-prey model is based on the assumption that depletion in a predator population has a reciprocal relationship with per capita availability of its preferred food. For the modified Leslie-Gower functional response in the case of severe scarcity, predator can switch over to other population (or alternative) but its growth will be limited by the fact that its most favorite food prey is not available in abundance.

The plan of this study is to analyze a dynamical model to guard forestry from the threats of toxicity and illegal logging and to increase forestry based industries. We have acquired an age structured forestry biomass via immature and mature forestry population considering that the industries are confined not to harvest pre-mature trees. Modified Leslie-Gower response function is also introduced as the alternative resource for industries when forestry resources are demolished.

\subsection{Basic Assumptions}

Modeling is an effective tool to develop the decision support to illuminate the real word problem. Consider the following assumptions for the proposed model to portrait system dynamics:

i) Forestry structural population, i.e., trees are classified via immature $\left(x_{1}\right)$, mature trees $\left(x_{2}\right)$ and industrialization $\left(x_{3}\right)$ i.e.

ii) Consider the logistic growth rate for immature trees with fixed growth rate and carrying capacity.

iii) Alternative is provided to industries for the conservation of forest biomass.

iv) Industries are used forestry based mature trees.

v) Consider the natural depletion rate for mature trees because of natural calamity.

vi) Consider the external toxicity which affect the immature trees.

vii) Illegal logging decreases the biomass of mature trees.

viii) Harvesting Effort is developed to harvest the mature trees.

ix) The total revenue is earned from the difference of pricing and harvesting cost applied to harvest mature forestry trees.

The conceptual model system for the schematic flow diagram is shown in figure below:

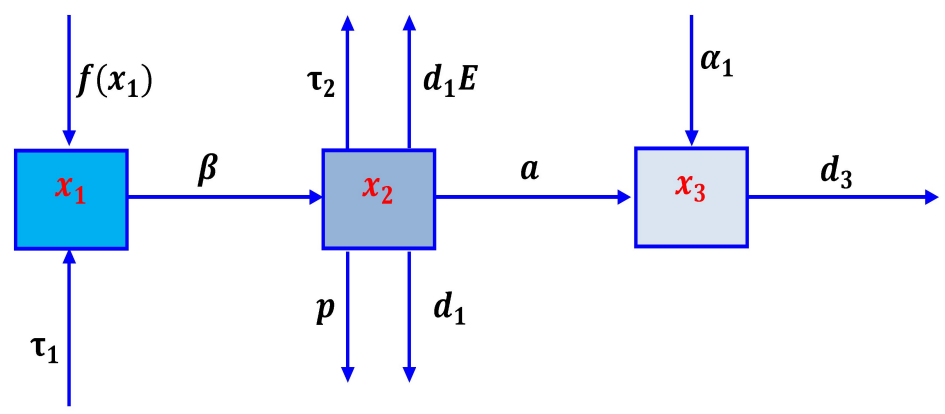

Figure 1: Schematic diagram

\section{Mathematical Model Formulation}

A simple age-structured forestry biomass model via immature (Juvenile) trees $\left(x_{1}\right)$, mature (adult) trees $\left(x_{2}\right)$ and number of industries (industrialization) $x_{3}$, the function $f\left(x_{1}\right)$ is a combination of logistic growth and new plantation for immature trees defined by $f_{1}\left(x_{1}\right)=r x_{1}\left(1-\frac{x_{1}}{k}\right)+\gamma x_{1}$, the function $f_{2}\left(x_{1}, x_{2}\right)$ is the modified Leslie-Gower term for mature trees and industrialization defined by $f_{3}\left(x_{2}, x_{3}\right)=x_{3}\left(\alpha_{1}-\frac{\alpha_{2} x_{3}}{a+x_{2}}\right)$ 
Journal of Nepal Mathematical Society (JNMS), Vol. 2, Issue 1 (2019); N. Hasan, H. A. Biswas, S. Uddin and $E$ is the effort expanded to harvest mature trees then the proposed dynamical system becomes [17]:

$$
\begin{aligned}
& \frac{d x_{1}}{d t}=r x_{1}\left(1-\frac{x_{1}}{k}\right)-\beta x_{1}+\gamma x_{1}, \\
& \frac{d x_{2}}{d t}=e \beta x_{1}-d_{1} E x_{2}-d_{2} x_{2}, \\
& \frac{d x_{3}}{d t}=\left(\alpha_{1}-\frac{\alpha_{2} x_{2}}{a+x_{2}}\right) x_{2}-d_{2} x_{3}, \\
& x_{1}(0) \geq 0, \quad x_{2}(0)>0, \quad x_{3}(0) \geq 0 .
\end{aligned}
$$

The ecological meaning of the system parameters are provided in a table formate.

Table 4.1: Description of parameters

\begin{tabular}{cl}
\hline Parameter & \multicolumn{1}{c}{ Description } \\
\hline$x_{1}$ & Immature trees (Juvenile) \\
$x_{2}$ & Mature trees (Adult) \\
$x_{3}$ & Industrialization (Number of Industries) \\
$r$ & Intrinsic growth rate of immature trees \\
$k$ & Rate of toxicity for immature trees \\
$\tau_{1}$ & Rate of toxicity for mature trees \\
$\tau_{2}$ & Rate of new plantation \\
$\gamma$ & Transition rate from immature to mature \\
$\beta$ & Measures the reduction rate of industrialization \\
$\alpha_{1}$ & Maximum value for the reduced rate of industries can attain \\
$\alpha_{2}$ & Average forestry protection rate \\
1 & Depletion rate of mature trees \\
$a$ & Natural depletion rate of mature trees \\
$d_{1}$ & Decreasing rate rate of industries in the absence of preferred mature trees \\
$d_{3}$ & Illegal logging of mature trees \\
$p$ & Harvesting effort \\
$E$ & Rate of conversion \\
$e$ &
\end{tabular}

Since our main objective is to examine the dynamical behavior of mangrove forest in the Sundarban ecosystem in the presence of harvesting, toxicity and illegal logging, then we have formulated the following dynamical model system:

$$
\begin{aligned}
& f_{1}=\frac{d x_{1}}{d t}=r x_{1}\left(1-\frac{x_{1}}{k}\right)-\beta x_{1}+\gamma x_{1}-\tau_{1} x_{1}^{3}, \\
& f_{2}=\frac{d x_{2}}{d t}=e \beta x_{1}-d_{1} E x_{2}-d_{2} x_{2}-p x_{2}-\tau_{2} x_{2}^{2}, \\
& f_{3}=\frac{d x_{3}}{d t}=\left(\alpha_{1}-\frac{\alpha_{2} x_{3}}{a+x_{2}}\right) x_{3}-d_{3} x_{3}, \\
& x_{1}(0) \geq 0, \quad x_{2}(0)>0, \quad x_{3}(0) \geq 0 .
\end{aligned}
$$

The term $\tau_{1} x_{1}^{3}$ can be interpreted as a type of functional response of the immature trees and comes directly to affect them by some external toxic substances released from the industries and water vehicles. Since $\frac{d}{d x_{1}}\left(\tau_{1} x_{1}^{3}\right)=3 \tau_{1} x_{1}^{2}>0$ and $\frac{d}{d x_{1}}\left(3 \tau_{1} x_{1}^{2}\right)=6 \tau_{1} x_{1}>0$ which is accelerated growth in the production of the toxic substance to the density of immature trees $x_{1}$. Also the similar case for mature trees $x_{2}$ except the effect of toxicity on the mature trees being less than on the immature trees is taken as $\tau_{2} x_{2}^{2}$ where $\tau_{2}$ be the coefficient of toxicity to the mature trees $\left(0<\tau_{2}<\tau_{1}<1\right)$. 
An Ecological Model for Sustainable Forest Management of Eco-system Based on Optimal Control Theory

\section{Preliminary Results}

Here, we will apply the following lemmas to establish the positivity, the boundedness and the permanence of the proposed model system.

Lemma 3.1. [6] If $a, b>0$ and $\frac{d X}{d t} \leq(\geq) X(t)(a-b X(t))$ with $X(t)>0$, then $\lim _{t \rightarrow+\infty} \sup X(t) \leq$ $\left(\lim _{t \rightarrow \infty} \inf X(t) \geq \frac{a}{b}\right)$.

Lemma 3.2. If $a, b>0$ and $\frac{d X}{d t} \leq(\geq) X(t)(a-b X(t))$ with $X(t)>0$. then for all $t \geq 0, X(t) \leq \frac{a}{b-C e^{-a t}}$ with $C=b-\frac{a}{X(0)}$. In particular $X(t) \leq \max \left\{X(0), \frac{a}{b}\right\}$ for all $t \geq 0$.

\subsection{Boundedness}

Theorem 3.1. All the solutions $\left(x_{1}(t), x_{2}(t), x_{3}(t)\right)$ of the system (4)-(6) in $R_{+}^{3}$ are always positive i.e., $x_{1}(t)>0, x_{2}(t)>0, x_{3}(t)>0$ for all $t \geq 0$.

Proof. The theorem is true since,

$$
\begin{aligned}
& x_{1}(t)=x_{1}(0) \exp \int_{0}^{t}\left[r\left(1-\frac{x_{1}(s)}{k}\right)-\beta+\gamma-\tau_{1} x_{1}^{2}(s)\right] d s \\
& x_{2}(t)=x_{2}(0) \exp \int_{0}^{t}\left[e \beta \frac{x_{1}(s)}{x_{2}(s)}-\left(d_{1} E+d_{2}+p+\tau_{2} x_{2}(s)\right)\right] d s \\
& x_{3}(t)=x_{3}(0) \exp \int_{0}^{t}\left[\alpha_{1}-d_{3}-\frac{\alpha_{2} x_{3}(s)}{a+x_{2}(s)}\right] d s
\end{aligned}
$$

Before analyzing the model system let us prove that the solutions to the system (4) - (6) corresponding the following theorem.

Theorem 3.2. All the solutions of the model system (4)-(6) with initial conditions are always bounded, for all $t \geq 0$.

Proof. From equation (4), we can write

$$
\frac{d x_{1}}{d t} \leq r x_{1}\left(1-\frac{x_{1}}{k}\right)+\gamma x_{1}
$$

From Lemma 3.2, we have

$$
X(t) \leq \max \left\{x_{1}(0), \frac{(r+\gamma) k}{r}\right\} \equiv M_{1} \quad \text { for all } \quad t \geq 0 .
$$

Further, from equation (5), we have

$$
\frac{d x_{2}}{d t}=e \beta x_{1}-d_{1} E x_{2}-d_{2} x_{2}-p x_{2} \leq e \beta M_{1}-d_{1} E x_{2}-d_{2} x_{2}-p x_{2}
$$

Again From Lemma 3.2, we have

$$
x_{2}(t) \leq \max \left\{x_{2}(0), \frac{\beta M_{1}}{d_{1} E+d_{2}+p}\right\} \equiv M_{2} \quad \text { for all } \quad t \geq 0
$$

Further, from equation (5), we have

$$
\frac{d x_{3}}{d t}=x_{3}\left(\alpha_{1}-\frac{\alpha_{2} x_{3}}{a+x_{2}}\right) \leq x_{3}\left(\alpha_{1}-\frac{\alpha_{2} x_{3}}{a+M_{2}}\right)
$$

Again, from Lemma 3.2, we have

$$
x_{3} \leq \max \left\{x_{3}(0), \frac{\alpha_{1}\left(a+M_{2}\right)}{\alpha_{2}}\right\} \equiv M_{3} \text { for all } t \geq 0 .
$$

The proof is complete for the boundedness of the model system and hence the system under the consideration is dissipative. 
Journal of Nepal Mathematical Society (JNMS), Vol. 2, Issue 1 (2019); N. Hasan, H. A. Biswas, S. Uddin

\subsection{Permanence}

Theorem 3.3. The system of equation (4) - (6) with initial conditions is permanent if $\lim _{t \rightarrow \infty} \sup \left(x_{1}\right) \leq M_{1}, \quad \lim _{t \rightarrow \infty} \sup \left(x_{2}\right) \leq M_{2}, \quad$ and $\quad \lim _{t \rightarrow \infty} \sup \left(x_{3}\right) \leq M_{3}$.

Proof. It is clear that from the equation (4) and Lemma 3.1, $0<x_{1}(t)<\frac{(r+\gamma)}{r}$, from equation (5) and Lemma 3.1, $x_{2} \leq \frac{e \beta M_{1}}{d_{1} E+d_{2}+p}$ and from equation (6) and lemma 3.1, $0<x_{3}(t)<\frac{\alpha_{1}\left(a+M_{2}\right)}{\alpha_{2}}$ for sufficiently large $t$. Hence, from the equation (6) we have,

$$
\frac{d x_{1}}{d t} \geq r x_{1}\left(1-\frac{x_{1}}{k}-\beta x_{1}\right) \text { or } \frac{(r-\beta) k}{r} \equiv N_{1}
$$

and then applying Lemma 3.1, we have,

$$
\lim _{t \rightarrow \infty} x_{1}(t) \geq N_{1}
$$

Further, from equation (5) we have,

$$
\frac{d x_{2}}{d t} \geq e \beta N_{1}-d_{1} E x_{2}-d_{2} x_{2}-p x_{2}
$$

, which gives by using Lemma 1

$$
\lim _{t \rightarrow \infty} i n f x_{2}(t) \geq \frac{\beta N_{1}}{d_{1} E+d_{2}+p_{1}} \equiv N_{2} .
$$

Further, from equation (6) we have,

$$
\frac{d x_{3}}{d t}=x_{3}\left(\alpha_{1}-\frac{\alpha_{2}}{a+N_{2}}\right)
$$

and then applying Lemma 3.1 we have,

$$
\lim _{t \rightarrow \infty} \inf x_{3}(t) \geq \frac{\left(\alpha_{1}-d_{2}-p\right)\left(a+N_{2}\right)}{\alpha_{2}} .
$$

Also, from inequalities (7)-(9), together with Lemma 3.1, we can write

$$
\lim _{t \rightarrow \infty} \sup \left(x_{1}\right) \leq M_{1}, \lim _{t \rightarrow \infty} \sup \left(x_{2}\right) \leq M_{2}, \quad \text { and } \quad \lim _{t \rightarrow \infty} \sup \left(x_{3}\right) \leq M_{3} .
$$

Now, taking $C_{1}=\min \left(N_{1}, N_{2}, N_{3}\right)$ and $C_{2}=\max \left(M_{1}, M_{2}, M_{3}\right)$, we can see the permanence for the model system.

\section{Equilibrium Analysis}

In this section, we establish the conditions for the existence of the four equilibrium points of the model system namely $E_{0}\left(x_{10}, x_{20}, x_{30}\right), \quad E_{1}\left(x_{11}, x_{21}, x_{31}\right), \quad E_{2}\left(x_{12}, x_{22}, x_{32}\right)$ and $E_{3}\left(x_{13}, x_{23}, x_{33}\right)$.

(a) Trivial equilibrium point: When the mature. immature and number of industries do not exists i.e., $x_{1}=x_{2}=x_{3}=0$ thus the equilibrium point is obtained $E_{0}\left(x_{10}, x_{20}, x_{30}\right)=E_{0}(0,0,0)$

(b) Boundary equilibrium point: When forest ecological biomass exist and industrialization does not exist i.e., $x_{1}=x_{2} \neq 0$ and $x_{3}=0$, then from the system (4)-(6), we get

$$
\begin{aligned}
& \frac{d x_{1}}{d t}=0 \\
\Rightarrow \quad & r x_{1}\left(1-\frac{x_{1}}{k}\right)+\gamma x_{1}-\beta x_{1}-3 \tau_{1} x_{1}^{2}=0 \\
\Rightarrow & x_{1}=\frac{-r \pm \sqrt{\left.\left(\frac{r}{k}\right)^{2}-4 \tau_{1}(\beta-\gamma-r)\right)}}{2 \tau_{1}}
\end{aligned}
$$



since the population density $x_{1}=\frac{-r-\sqrt{\left.\left(\frac{r}{k}\right)^{2}-4 \tau_{1}(\beta-\gamma-r)\right)}}{2 \tau_{1}}$ which is biologically meaningless. So

$$
\begin{aligned}
& r^{2}-4 k^{2} \tau_{1}(\beta-r-\gamma)>0 \\
\Rightarrow \quad & r^{2}>4 k^{2} \tau_{1}(\beta-r-\gamma)
\end{aligned}
$$

and

$$
\begin{aligned}
& \frac{d x_{2}}{d t}=0 \\
\Rightarrow \quad & e \beta x_{1}-d_{1} E x_{2}-d_{2} x_{2}-p x_{2}-\tau_{2} x_{2}^{2}=0 \\
\Rightarrow & x_{2}=\frac{e \beta x_{1}^{*}}{r\left(d_{1} E+d_{2}+p\right)}
\end{aligned}
$$

where

$$
x_{1}^{*}=\frac{-r-\sqrt{\left.\left(\frac{r}{k}\right)^{2}-4 \tau_{1}(\beta-\gamma-r)\right)}}{2 \tau_{1}}
$$

and the axial equilibria of the system exists when $r+\gamma>\beta$.

Therefore, the equilibrium point is obtained

$$
E_{1}\left(x_{11}, x_{21}, 0\right)=E_{1}\left(\frac{k}{r}(r+\gamma-\beta), \frac{\beta k(r+\gamma-\beta)}{r\left(d_{1} E+d_{2}+p_{1}\right)}, 0\right) .
$$

Thus, in the absence of industrialization $x_{3}$, the total intrinsic growth rate of immature trees and new plantation must be greater than its transition rate from immature to mature for the point $E_{1}\left(x_{11}, x_{21}, 0\right)$ to exists.

(c) Axial equilibrium point: Where there are no immature and mature trees i.e., $x_{1}=x_{2}=0$ and industrialization $x_{3} \neq 0$ then from the system $(2.6)$ we get,

$$
\begin{aligned}
& \frac{d x_{3}}{d t}=0 \\
\Rightarrow & x_{3}\left(\alpha_{1}-\frac{\alpha_{2} x_{3}}{a}-d_{3}\right)=0 \\
\Rightarrow & x_{3}=\frac{a}{\alpha_{2}}\left(\alpha_{1}-d_{3}\right) .
\end{aligned}
$$

Thus, the equilibrium point is obtained $E_{2}\left(0,0, x_{32},\right)=E_{2}\left(0,0, \frac{a}{\alpha_{2}}\left(\alpha_{1}-d_{3}\right)\right)$.

This result shows that the model assumption exists when $\alpha_{1}>d_{3}$ i.e., the growth rate of industrialization must always be greater than the depletion of industries.

(d) Interior equilibrium point: When all the state variables of the model system are co-exists i.e., $x_{1} \neq$ $0, x_{2} \neq 0, x_{3} \neq 0$ then from the system(2.4)-(2.6) we have, $\frac{d x_{1}}{d t}=0, \frac{d x_{2}}{d t}=0$ and $\frac{d x_{3}}{d t}=0$

i.e., $x_{1}=\frac{k}{r}(r+\gamma-\beta), \quad x_{2}=\frac{e \beta k(r+\gamma-\beta)}{r\left(d_{1} E+d_{2}+p\right)}$ and $x_{3}=\frac{a}{\alpha_{2}}\left(\alpha_{1}-d_{3}\right)$.

The interior equilibrium point $E_{3}\left(x_{13}, x_{23}, x_{33}\right)$ i.e., $\left(\frac{k}{r}(r+\gamma-\beta), \frac{e \beta(r+\gamma-\beta)}{r\left(d_{1} E+d_{2}+p_{1}\right)}, \frac{a}{\alpha_{2}}\left(\alpha_{1}-d_{3}\right)\right)$ exists only when $r+\gamma>\beta$ and $\alpha_{1}>d_{3}$.

\section{Dynamic Behavior}

\subsection{Local Stability Analysis}

In this section, we analyze the stability properties of the equilibrium points $E_{0}, E_{1}, E_{2}$ and $E_{3}$. The local stability is established through Jacobian matrix of the system and finding the eigenvalues to evaluate at each equilibrium point. For model system the Jacobian matrix is given by 


$$
\left[\begin{array}{lll}
\frac{\partial f_{1}}{\partial x_{1}} & \frac{\partial f_{1}}{\partial x_{2}} & \frac{\partial f_{1}}{\partial x_{3}} \\
\frac{\partial f_{2}}{\partial x_{1}} & \frac{\partial f_{2}}{\partial x_{2}} & \frac{\partial f_{2}}{\partial x_{3}} \\
\frac{\partial f_{3}}{\partial x_{1}} & \frac{\partial f_{3}}{\partial x_{2}} & \frac{\partial f_{3}}{\partial x_{3}}
\end{array}\right]
$$

For the model system (4)-(6), its corresponding Jacobian matrix is

$$
J\left(E_{i}\right)=\left[\begin{array}{lll}
J_{11} & J_{12} & J_{13} \\
J_{21} & J_{22} & J_{23} \\
J_{31} & J_{32} & J_{33}
\end{array}\right]
$$

where

$$
\begin{aligned}
& J_{11}=r+\gamma-\beta-\frac{2 x_{1} r}{k}-3 \tau_{1} x_{1}^{2} \\
& J_{12}=0 \\
& J_{13}=0 \\
& J_{21}=e \beta \\
& J_{22}=-d_{1} E-d_{2}-p \\
& J_{23}=0 \\
& J_{31}=0, \\
& J_{32}=\frac{\alpha_{2} x_{3}^{2}}{\left(a+x_{2}\right)^{2}}, \\
& J_{33}=\alpha_{1}-d_{3}-\frac{2 x_{3} \alpha_{2}}{a+x_{2}} .
\end{aligned}
$$

\subsubsection{Behavior of the system around $E_{0}(0,0,0)$ :}

The Jacobian matrix $J_{0}$ at $E_{0}(0,0,0)$ is

$$
J\left(E_{0}\right)=\left[\begin{array}{ccc}
r-\beta+\gamma & 0 & 0 \\
\beta & -\left(d_{1} E+d_{2}+p\right) & 0 \\
0 & 0 & \alpha_{1}-d_{3}
\end{array}\right]
$$

The eigenvalues of the Jacobian matrix $J_{0}$ at $E_{0}$ are $r+\gamma_{\beta},-\left(d_{1} E-d_{2}-p\right)$ and $\alpha_{1}-d_{3}$. Hence, the equilibrium point $E_{0}(0,0,0)$ is asymptotically stable if $\beta>r+\gamma$ and $d_{3}>\alpha_{1}$.

\subsubsection{Behavior of the system around $E_{1}\left(x_{11}, 0,0\right)$ :}

The Jacobian matrix $J_{0}$ at $E_{1}\left(x_{11}, 0,0\right)$ is

$$
J\left(E_{1}\right)=\left[\begin{array}{ccc}
r-\beta+\gamma-\frac{r x_{11}}{k}-3 \tau_{1} x_{11}^{2} & 0 & 0 \\
e \beta & -\left(d_{1} E+d_{2}+p\right) & 0 \\
0 & 0 & \alpha_{1}-d_{3}
\end{array}\right]
$$

The eigenvalue of the Jacobian matrix at $E_{1}\left(x_{11}, x_{21}, 0\right)$ are

$$
\lambda=r-\frac{r x_{11}}{k}-\beta+\gamma-3 \tau_{1} x_{11}, \lambda=-\left(d_{1} E+d_{2}+p\right) \text { and } \lambda=\alpha_{1}-d_{3}
$$

$\lambda$ is negative $r+\gamma<\left(\beta+\frac{r x_{11}}{k}+3 \tau_{1} x_{11}^{2}\right)$ and $\alpha_{1}<d_{3}$. Since all the eigenvalues are negative, hence the equilibrium point $E_{1}\left(x_{1}, x_{2}, 0\right)$ is locally asymptotically stable. 


\subsubsection{Behavior of the system around $E_{2}\left(0,0, x_{3}\right)$ :}

The Jacobian matrix $J_{2}$ at $E_{2}\left(0,0, x_{3}\right)$ is

$$
J\left(E_{2}\right)=\left[\begin{array}{ccc}
r-\beta+\gamma & 0 & 0 \\
e \beta & -\left(d_{1} E+d_{2}+p\right) & 0 \\
0 & \frac{\alpha_{2} x_{33}^{2}}{a^{2}} & \alpha_{1}-d_{3}-\frac{\alpha_{2} x_{32}}{a}
\end{array}\right]
$$

The eigen values of the Jacobian matrix at $E_{2}\left(0,0, x_{33}\right)$ are $\lambda=r-\beta+\gamma, \quad \lambda=-\left(d_{1} E+d_{2}+p\right)$ and $\lambda=\alpha_{1}-d_{3}-\frac{\alpha_{2} x_{3}}{a}$. Therefore $\lambda$ is negative when $\left.\alpha_{1}<d_{3}+\frac{\alpha_{2} x_{3}}{a}\right)$ and $r+\gamma<\beta$. Since all the eigenvalues are negative, hence the equilibrium point $E_{1}\left(0,0, x_{33}\right)$ is locally asymptotically stable.

\subsubsection{Behavior of the system around $\left.E_{3}\left(x_{13}, x_{23}, x_{33}\right)\right)$ :}

The Jacobian matrix $J_{3}$ at the co-existence point $E_{3}\left(x_{13}, x_{23}, x_{33}\right)$ :

$$
J\left(E_{3}\right)=\left[\begin{array}{ccc}
r-\beta+\gamma-\frac{2 r x_{1}}{k}-3 \tau_{1} x_{13} & 0 & 0 \\
e \beta & -\left(d_{1} E+d_{2}+p+2 \tau_{2} x_{2}\right) & 0 \\
0 & \frac{\alpha-2 x_{33}^{2}}{\left(a+x_{23}\right)^{2}} & \alpha_{1}-d_{3}-\frac{\alpha_{2} x_{33}}{a+x_{23}}
\end{array}\right]
$$

Eigenvalues corresponding to the point $E_{3}\left(x_{13}, x_{23}, x_{33}\right)$ are the roots of the equation

$$
\lambda^{3}+A_{1} \lambda^{2}+A_{2} \lambda+A_{3}=0
$$

where,

$$
\begin{aligned}
& A_{1}=-C_{11}-C_{22}-C_{33} \\
& A_{2}=C_{22} C_{33}+C_{11} C_{22}+C_{11} C_{33}-C_{12} C_{21}-C_{23} C_{32} \\
& A_{3}=C_{12} C_{21} C_{33}+C_{11} C_{23} C_{32}-C_{11} C_{22} C_{33}
\end{aligned}
$$

i.e.,

$$
\begin{aligned}
A_{1}= & \frac{2 r x_{1}}{k}+\frac{2 \alpha x_{3}}{a+x_{2}}+e \beta+d_{1} E+d_{2}+d_{3}+p+3 \tau_{1} x_{13}+\frac{\alpha_{2} x_{33}}{a+x_{23}}-r-\gamma-\alpha_{1}, \\
A_{2}= & \left(d_{1} E+d_{2}+p-2 \tau_{2} x_{2}\right)\left(\frac{\alpha_{2} x_{33}}{a+x_{23}}+d_{3}-\alpha_{1}\right)-\left(r+\beta-\gamma \frac{2 r x_{1}}{k}-3 \tau_{1} x_{13}\right)\left(d_{1} E+d_{2}+p+2 \tau_{2} x_{23}\right) \\
& +\left(r+\beta-\gamma \frac{2 r x_{1}}{k}-3 \tau_{1} x_{13}\right)\left(\alpha_{1}-d_{3}-\frac{2 \alpha_{2} x_{3}}{a+x_{2}}\right) \\
A_{3}= & \left(r+\gamma-\beta-\frac{2 r x_{1}}{k}\right)\left(d_{1} E+d_{2}+p+2 \tau_{2} x_{23}\right)\left(\alpha_{1}-\frac{2 \alpha_{2} x_{3}}{\left(a+x_{2}\right)}\right) .
\end{aligned}
$$

From Routh-Hurwitz criterion, $E_{3}$ is locally asymptotically stable, if and only if

$$
A_{1}>0, A_{3}>0, A_{1} A_{2}>A_{3}
$$

Now,

$$
\begin{aligned}
A_{1} A_{2}-A_{3}= & -\left(C_{11}\right)^{2} C_{22}-\left(C_{11}\right)^{2} C_{33}+C_{11} C_{12} C_{21}-\left(C_{22}\right)^{2} C_{33}-\left(C_{22}\right)^{2} C_{11}-2 C_{11} C_{22} C_{33} \\
& +C_{22} C_{13} C_{21}+C_{23} C_{32} C_{22}-\left(C_{33}\right)^{2} C_{22}-C_{11}\left(C_{33}\right)^{2}+C_{23} C_{32} C_{33}
\end{aligned}
$$

as $C_{12}<0, \quad C_{21}>0, \quad C_{23}<0, \quad C_{32}>0, \quad C_{33}<0$.

It is easy to examine that

$$
A_{1} A_{2}-A_{3}>0 \text { if } C_{11}<0, C_{22}<0
$$



i.e.,

$$
\begin{aligned}
& r+\gamma<\frac{2 r x_{13}}{k}+\beta+3 \tau_{1} x_{13}^{2}, \\
& \text { and } \quad \alpha_{1}<d_{3}+\frac{2 \alpha_{2} x_{33}}{a+x_{23}} .
\end{aligned}
$$

which satisfies the Routh-Hurwitz criteria. Hence we can conclude that the interior equilibrium is locally asymptotically stable.

\subsection{Global Stability:}

To show the global stability of the system (4)-(6) we consider

$$
\mathfrak{R}_{+}^{3}=\left[\left(x_{1}, x_{2}, x_{3}\right) \in \mathfrak{R}_{+}^{3}, x_{1} \leq 0, x_{2} \leq 0, x_{3} \leq 0\right]
$$

and the function $J\left(E_{3}\right): \mathfrak{R}_{+}^{3} \rightarrow \mathfrak{R}^{3}$ then construct a Lyanpnov-Lasaile's function as follows,

$$
J\left(E_{3}\right)=L_{1}\left(x_{1}-x_{13}-x_{13} \ln \left(x_{1}\right)+L_{2}\left(x_{2}-x_{23}-x_{23} \ln \left(x_{2}\right)\right)+L_{3}\left(x_{3}-x_{33}-x_{33} \ln \left(x_{3}\right)\right)\right.
$$

where $L_{1}, L_{2}$ and $L_{3}$ be positive constants to be determined. We can easily verify that the function $L\left(E_{3}\right)=0$ at $E_{3}\left(x_{13}, x_{23}, x_{33}\right)$ and is positive for all other values of $x_{1}, x_{2}$ and $x_{3}$. Then the time derivative of $L\left(E_{3}\right)$ along the solution of the system is given by,

$$
\begin{aligned}
\frac{d L}{d t} & =L_{1}\left(1-\frac{x_{13}}{x_{1}}\right) \frac{d x_{1}}{d t}+L_{2}\left(1-\frac{x_{23}}{x_{2}}\right) \frac{d x_{2}}{d t}+L_{3}\left(1-\frac{x_{33}}{x_{3}}\right) \frac{d x_{3}}{d t} \\
& =L_{1}\left(x_{1}-x_{13}\right)\left\{r\left(1-\frac{x_{1}}{k}+\gamma-\beta-3 \tau_{1} x_{1}^{2}\right\}+L_{2}\left(x_{2}-x_{23}\right)\left\{\frac{e \beta x_{1}}{x_{2}}-d_{1} E-d_{2}-p-2 \tau_{2} x_{2}\right\}\right. \\
& +L_{3}\left(x_{3}-x_{33}\left\{\alpha_{1}-\frac{\alpha_{2} x_{3}}{a+x_{2}}-d_{3}\right\} .\right.
\end{aligned}
$$

At the equilibrium point $E_{3}$, we have,

$$
\begin{aligned}
r+\gamma-\beta & =\frac{r x_{13}}{k}+3 \tau_{1} x_{13}^{2}, \\
d_{1} E+d_{2}+p & =\frac{\beta x_{13}}{x_{23}}-2 \tau_{2} x_{23}, \\
\alpha_{1}-d_{3} & =\frac{\alpha_{2} x_{33}}{a+x_{23}} .
\end{aligned}
$$

Thus the equation (13) becomes,

$$
\begin{aligned}
\frac{d L}{d t}= & L_{1}\left(x_{1}-x_{13}\right)\left\{r+\gamma-\beta-\frac{r x_{1}}{k}\right\}+L_{2}\left(x_{2}-x_{23}\right)\left\{\frac{e \beta x_{1}}{x_{2}}-\left(d_{1} E+d_{2}+p\right)\right\} \\
& +L_{3}\left(x_{3}-x_{33}\left\{\alpha_{1}-d_{3}-\frac{\alpha_{2} x_{3}}{a+x_{2}}\right\}\right. \\
\frac{d L}{d t}= & L_{1}\left(x_{1}-x_{13}\right)\left\{\frac{r x_{13}}{k}-\frac{r x_{1}}{k}\right\}+L_{2}\left(x_{2}-x_{23}\right)\left\{\frac{e \beta x_{1}}{x_{2}}-\left(d_{1} E+d_{2}+p\right)\right\} \\
& +L_{3}\left(x_{3}-x_{33}\right)\left\{\frac{\alpha_{2} x_{33}}{a+x_{2}}-\frac{\alpha_{2} x_{3}}{a+x_{2}}\right\} \\
\frac{d L}{d t}= & -L_{1}\left(x_{1}-x_{13}\right)^{2} \frac{r}{k}+L_{2}\left(x_{2}-x_{23}\right)\left\{\frac{e \beta x_{1}}{x_{2}}-\left(d_{1} E+\alpha_{2}+p\right)\right\} \\
& -L_{3}\left(x_{3}-x_{33}\right)^{2} \frac{\alpha_{2}}{a+x_{2}} . \\
& \frac{d L\left(E_{3}\right)}{d t} \leq L_{2} \beta\left(x_{2}-x_{23}\right)\left(\frac{x_{1}}{x_{2}}-\frac{x_{13}}{x_{23}}\right)
\end{aligned}
$$



Let $\quad L_{2}=\frac{1}{\beta}$

Then,

$$
\begin{aligned}
\frac{d L\left(E_{3}\right)}{d t} & \leq L_{2}\left(x_{2}-x_{23}\right)\left(\frac{x_{1}}{x_{2}}-\frac{x_{13}}{x_{23}}\right) \\
& \Rightarrow \frac{d L\left(E_{3}\right)}{d t} \leq 0
\end{aligned}
$$

If $x_{2} \geq x_{23}$ and $x_{1} \geq x_{13}$.

Again $\frac{d L\left(E_{3}\right)}{d t}=0$ when $\left(x_{1}, x_{2}, x_{3}\right)=\left(x_{13}, x_{23}, x_{33}\right)$ which satisfies the Lyapnove-Lasalle's principle.

Hence $E_{3}$ globally asymptotically stable.

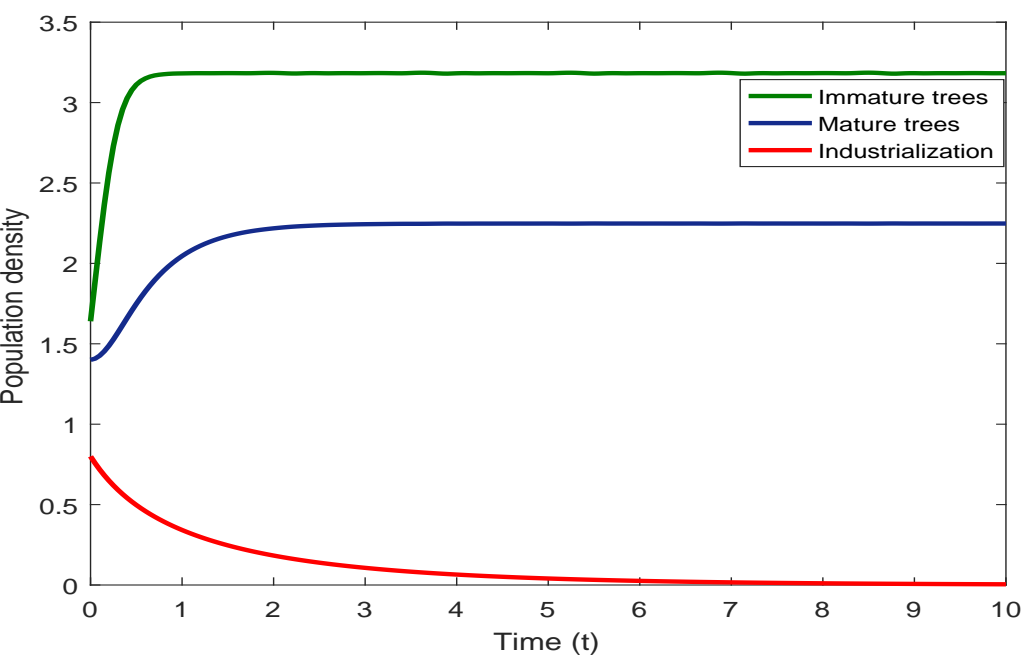

(a)

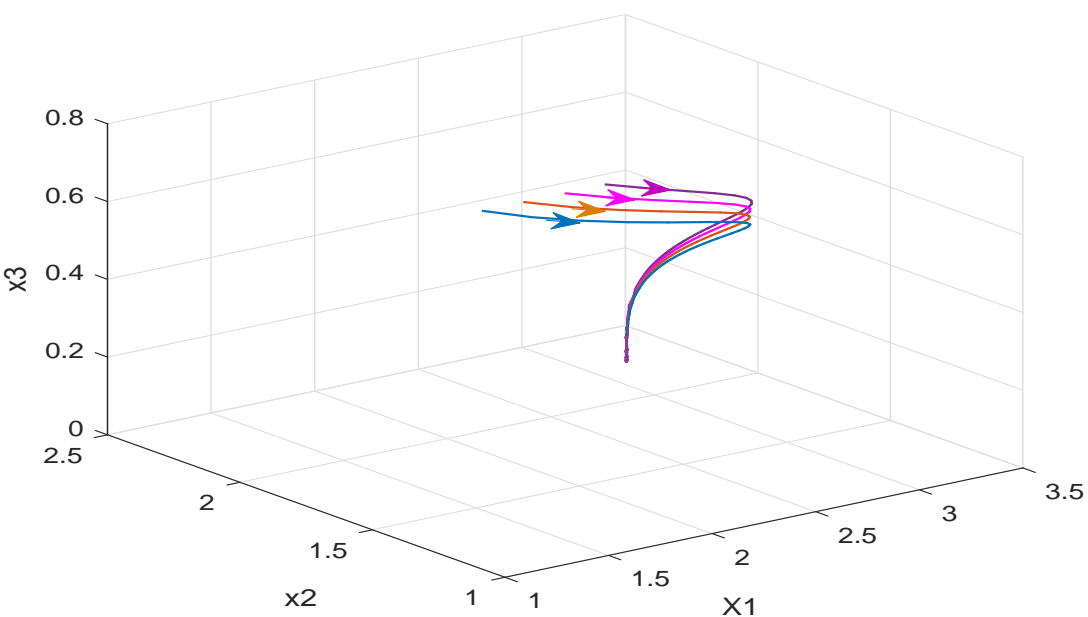

(b)

Figure 2: Global stability around the positive interior equilibrium point $E_{3}\left(x_{13}, x_{23}, x_{33}\right)$

\section{Bionomic Equilibrium}

The forestry based industries and the regulatory agency are two different component to the country at large then the earning revenues by them are collected to the country through the forestry. The net economic 
revenue to the country is

$$
\prod\left(x, E, p_{1}, t\right)=\left(d_{1} p_{1} x_{2}-c\right) E
$$

where $p_{1}$ is the per unit price of forestry biomass and $c$ is the constant harvesting cost per unit effort for the mature trees population.

This revenue is equals to the forestry based industries and the economic revenue to the agency, then the bionomic equilibrium $\left(x_{1 \infty}, x_{2 \infty}, x_{3 \infty}, E_{\infty}\right)$ is given by the following simultaneous equations,

$$
\begin{aligned}
r x_{1}\left(1-\frac{x_{1}}{k}\right)-\beta x_{1}+\gamma x_{1}-\tau_{1} x_{1}^{3} & =0, \\
e \beta x_{1}-d_{1} E x_{2}-d_{2} x_{2}-p x_{2}-\tau_{2} x_{2} & =0, \\
\left(\alpha_{1}-\frac{\alpha_{2} x_{3}}{a+x_{2}}\right)-d_{3} x_{3} & =0, \\
\prod=\left(d_{1} p_{1} x_{2}-c\right) E & =0 .
\end{aligned}
$$

By solving (16)-(19), we get

$$
\begin{aligned}
x_{1 \infty} & =\frac{1}{\beta}\left(d_{1} E+d_{2}+p+\frac{\tau_{2} c}{d_{1} p}\right), \\
x_{2 \infty} & =\frac{c}{d_{1} p_{1}}, \\
x_{3 \infty} & =\frac{\left(\alpha_{1}-d_{3}\right)\left(a d_{1} p_{1}+c\right)}{\alpha_{2} d_{1} x_{1}} \quad \alpha_{1}>d_{3}, \\
E_{\infty} & =\frac{1}{d_{1}^{2} p_{1}}\left(d_{1} d_{2} p_{1}+d_{1} p_{1}^{2}+\tau_{2} c\right)+\frac{e \beta d_{1} p_{1} r}{2 \tau_{1} k C}-\frac{e \beta d_{1} p_{1} r}{2 \tau_{1} k c} \sqrt{k^{2}+4 r k^{2}(r+\gamma-\beta)} .
\end{aligned}
$$

Considering the equation (23) we can say that,

$$
E_{\infty}>0 \text { when } 1+4 r(r+\gamma) \leq 4 r \beta .
$$

Hence we conclude that the bionomic equilibrium exists.

\section{Optimal Control Model}

The control of the system (4)-(6) is possible to do if there is a certain limit that still can be reached by human. Control that applied in the model system (4)-(6)) is in the form of reducing of toxicity and illegal logging. Based on that we can form the model with the control variables as follows:

$$
\begin{aligned}
\frac{d x_{1}}{d t} & =r x_{1}\left(1-\frac{x_{1}}{k}\right)-\beta x_{1}+\gamma x_{1}-\left(1-u_{1}\right) \tau_{1} x_{1}^{3}, \\
\frac{d x_{2}}{d t} & =e \beta x_{1}-d_{1} E x_{2}-d_{2} x_{2}-\left(1-u_{2}\right) p x_{2}-\left(1-u_{1}\right) \tau_{2} x_{2}^{2}, \\
\frac{d x_{3}}{d t} & =\left(\alpha_{1}-\frac{\alpha_{2} x_{2}}{a+x_{2}}\right) x_{2}-d_{3} x_{3},
\end{aligned}
$$

with

$$
x_{1}(0) \geq 0, \quad x_{2}(0)>0, \quad x_{3}(0) \geq 0, \quad u_{1}(0) \geq 0, \quad u_{2}(0) \geq 0 .
$$

The objective of the optimal control is to maximize the number of immature and mature trees and also minimize the cost of the controls.

The Pontryagin's maximum principle [19] the objective function is given by

$$
\operatorname{Maximize} J(x, u)=\left(B_{1} x_{1}(T)+B_{2} x_{2}(T)\right)-\int_{0}^{T}\left(A_{1} u_{1}^{2}+A_{2} u_{2}^{2}\right) d t .
$$


Subject to

$$
\begin{aligned}
\dot{x}(t) & =f(t, x, u) \forall t \in[0, T], \\
x(0) & =x_{0}, \\
u(t) \in U &
\end{aligned}
$$

where,

$$
\begin{aligned}
x(t) & =\left(x_{1}(t), x_{2}(t), x_{3}(t)\right), \\
x(0) & =\left(x_{10}, x_{20}, x_{30}\right), \\
u(t) & =\left(u_{1}(t), u_{2}(t)\right)
\end{aligned}
$$

and $U(t)=\left(u_{1}(t), u_{2}(t)\right) \mid 0 \leq u_{i}(t) \leq 1, i=1,2$ where $A_{1}, A_{2}, B_{1}, B_{2}$ are positive weight. The term $A_{1} u_{1}^{2}$ is the cost of control efforts on reducing toxicity and the term $A_{2} u_{2}^{2}$ is the cost control efforts on anti-logging strategy.

\subsection{Existence of an Optimal Control}

The existence of the optimal control pair can be obtained using a result by Fleming and Rishel ([22] Theorem 4.1, pp 68-69). We first show that the set of all feasible solution to the control problem is non-empty.

Theorem 7.1. Given $u \in U$ there exists a bounded solution to the initial value problem defined in (24) and (25).

Proof. The solutions to (24) and (25) are bounded below by the trivial solutions. An upper bound can be proved using the fact that a super solution $\left(x^{*}, u^{*}\right)$ satisfying

$$
\begin{aligned}
& \frac{d x_{1}^{*}}{d t}=r_{1} x_{1}+\gamma x_{1}+u_{1} \tau_{1} x_{1}^{3}, \\
& \frac{d x_{2}^{*}}{d t}=e \beta x_{1}+u_{1} p x_{2}+u_{1} \tau_{2} x_{2}^{2},
\end{aligned}
$$

are bounded on a finite time interval.

Theorem 7.2. Consider the control problem with system equations (24) and (25), there exists $u^{*}=\left(u_{1}^{*}, u_{2}^{*}\right) \in U$, such that

$$
\max _{u \in U} J(u)=J\left(u^{*}\right)
$$

Proof. In order to verify the condition to use the result from [21], first we note that the solutions are bounded.

Second, we use a result by Lukes ([20], Theorem 9.2.1, pp 182) to give the existence of solution of ODE's (24) and (25) with bounded co-efficients.

Note that $U$ is bounded and convex.

Since our state system is bilinear in $u_{1}, u_{2}$ and the RHS of (24) and (25) is continuous then it can be written as $\bar{f}(t, \bar{x}, \bar{u})=\bar{\alpha}(t, x)+\bar{\beta}(t, x) \bar{u}$, and the boundedness of solutions gives $|\bar{f}(t, \bar{x}, \bar{u})| \leq c_{3}(1+|\bar{x}|+|\bar{u}|)$ for $0 \leq t \leq T, \bar{x} \in R_{+}^{2}, \bar{u} \in R_{+}^{2}$ where $\bar{x}=\left(x_{1}, x_{2}\right)$ and $\bar{u}=\left(u_{1}, u_{2}\right)$.

In order to verify the convexity of $L$, the integrand of our objective function, we can show

$$
L(t, x,(1-\epsilon) \bar{u}, \epsilon \bar{v},(1-\epsilon) \bar{w}) \geq(1-\epsilon) L(t, \bar{x}, \bar{u})+\epsilon L(t, \bar{x}, \bar{v})+(1+\epsilon) L(t, \bar{x}, \bar{w}) \quad \text { for } \quad 0<\epsilon<1 \text {. }
$$


where

$$
\begin{aligned}
& L(t, \bar{x}, \bar{u})=x_{1}+x_{2}-\left(\gamma_{1} u_{1}^{2}+\gamma_{2} u_{2}^{2}\right) \\
& =x_{1}+x_{2}-\gamma_{1}\left((1-\epsilon) u_{1}+\epsilon v_{1}+(1+\epsilon) w_{1}\right)^{2} \\
& -\gamma_{2}\left((1-\epsilon) u_{2}+\epsilon v_{2}+(1+\epsilon) w_{2}\right)^{2} \\
& -\gamma_{3}\left((1-\epsilon) u_{3}+\epsilon v_{3}+(1+\epsilon) w_{3}\right)^{2} \\
& =x_{1}+x_{2}-\gamma_{1}\left((1-\epsilon)^{2} u_{1}^{2}+\epsilon^{2} v_{1}^{2}+(1+\epsilon)^{2} w_{1}^{2}+2 \epsilon(1-\epsilon) u_{1} v_{1}+2 \epsilon(1+\epsilon) v_{1} w_{1}\right. \\
& \left.+2\left(1-\epsilon^{2}\right) u_{1} w_{1}\right)-\gamma_{2}\left((1-\epsilon)^{2} u_{2}^{2}+\epsilon^{2} v_{2}^{2}+(1+\epsilon)^{2} w_{2}^{2}+2 \epsilon(1-\epsilon) u_{2} v_{2}+2 \epsilon(1+\epsilon) v_{2} w_{2}\right. \\
& \left.+2\left(1-\epsilon^{2}\right) u_{2} w_{2}\right)-\gamma_{3}\left((1-\epsilon)^{2} u_{3}^{2}+\epsilon^{2} v_{3}^{2}+(1+\epsilon)^{2} w_{3}^{2}+2 \epsilon(1-\epsilon) u_{3} v_{3}+2 \epsilon(1+\epsilon) v_{3} w_{3}\right. \\
& \left.+2\left(1-\epsilon^{2}\right) u_{3} w_{3}\right) \\
& =x_{1}+x_{2}-\gamma_{1}\left(\left(1-2 \epsilon+\epsilon^{2}\right) u_{1}^{2}+\epsilon^{2} v_{1}^{2}+\left(1+2 \epsilon+\epsilon^{2}\right) w_{1}^{2}+2 \epsilon(1-\epsilon) u_{1} v_{1}+2 \epsilon(1+\epsilon) v_{1} w_{1}\right. \\
& \left.+2\left(1-\epsilon^{2}\right) u_{1} w_{1}\right)-\gamma_{2}\left(\left(1-2 \epsilon+\epsilon^{2}\right) u_{2}^{2}+\epsilon^{2} v_{2}^{2}+\left(1+2 \epsilon+\epsilon^{2}\right) w_{2}^{2}+2 \epsilon(1-\epsilon) u_{2} v_{2}\right. \\
& \left.+2 \epsilon(1+\epsilon) v_{2} w_{2}+2\left(1-\epsilon^{2}\right) u_{2} w_{2}\right)-\gamma_{3}\left(\left(1-2 \epsilon+\epsilon^{2}\right) u_{3}^{2}+\epsilon^{2} v_{3}^{2}+\left(1+2 \epsilon+\epsilon^{2}\right) w_{3}^{2}\right. \\
& \left.+2 \epsilon(1-\epsilon) u_{3} v_{3}+2 \epsilon(1+\epsilon) v_{3} w_{3}+2\left(1-\epsilon^{2}\right) u_{3} w_{3}\right) \\
& =x_{1}+x_{2}-\left[\gamma_{1}\left(u_{1}^{2}+w_{1}^{2}\right)+\gamma_{2}\left(u_{2}^{2}+w_{2}^{2}\right)+\gamma_{3}\left(u_{3}^{2}+w_{3}^{2}\right)\right] \\
& -\gamma_{1}\left[\left(\epsilon^{2}-2 \epsilon+\right) u_{1}^{2}+\epsilon^{2} v_{1}^{2}+\left(\epsilon^{2}+2 \epsilon\right) w_{1}^{2}+2 \epsilon(1-\epsilon) u_{1} v_{1}+2 \epsilon(1+\epsilon) v_{1} w_{1}+2\left(1-\epsilon^{2}\right) u_{1} w_{1}\right] \\
& -\gamma_{2}\left[\left(\epsilon^{2}-2 \epsilon\right) u_{2}^{2}+\epsilon^{2} v_{2}^{2}+\left(\epsilon^{2}+2 \epsilon\right) w_{2}^{2}+2 \epsilon(1-\epsilon) u_{2} v_{2}+2 \epsilon(1+\epsilon) v_{2} w_{2}+2\left(1-\epsilon^{2}\right) u_{2} w_{2}\right] \\
& -\gamma_{3}\left[\left(\epsilon^{2}-2 \epsilon\right) u_{3}^{2}+\epsilon^{2} v_{3}^{2}+\left(\epsilon^{2}+2 \epsilon\right) w_{3}^{2}+2 \epsilon(1-\epsilon) u_{3} v_{3}+2 \epsilon(1+\epsilon) v_{3} w_{3}+2\left(1-\epsilon^{2}\right) u_{3} w_{3}\right) \\
& \text { and } \quad(1-\epsilon) L(t, \bar{x}, \bar{u})+\epsilon L(t, \bar{x}, \bar{v})+(1+\epsilon) L(t, \bar{x}, \bar{w}) \\
& =(1-\epsilon)\left[x_{1}+x_{2}-\left(\gamma_{1} u_{1}^{2}+\gamma_{2} u_{2}^{2}+\gamma_{3} u_{3}^{2}\right)\right] \\
& +\epsilon\left[x_{1}+x_{2}-\left(\gamma_{1} v_{1}^{2}+\gamma_{2} v_{2}^{2}+\gamma_{3} v_{3}^{2}\right)\right] \\
& +(1+\epsilon)\left[x_{1}+x_{2}-\left(\gamma_{1} w_{1}^{2}+\gamma_{2} w_{2}^{2}+\gamma_{3} w_{3}^{2}\right)\right] \\
& =x_{1}+x_{2}-\epsilon\left(-\gamma_{1} u_{1}^{2}-\gamma_{2} u_{2}^{2}-\gamma_{3} u_{3}^{2}+\gamma_{1} v_{1}^{2}+\gamma_{2} v_{2}^{2}+\gamma_{3} v_{3}^{2}-\gamma_{1} w_{1}^{2}-\gamma_{2} w_{2}^{2}-\gamma_{3} w_{3}^{2}\right) \text {. }
\end{aligned}
$$

Thus, in order to show that $L(t, x,$.$) is convex in U$, we note that the following inequality holds:

$$
\begin{aligned}
& \gamma_{1}\left[\left(\epsilon^{2}-2 \epsilon+\right) u_{1}^{2}+\epsilon^{2} v_{1}^{2}+2 \epsilon(1-\epsilon) u_{1} v_{1}\right]-\gamma_{2}\left[\left(\epsilon^{2}-2 \epsilon\right) u_{2}^{2}+\epsilon^{2} v_{2}^{2}+\left(\epsilon^{2}+2 \epsilon(1-\epsilon) u_{2} v_{2}\right]\right. \\
& \leq \epsilon\left(-\gamma_{1} u_{1}^{2}-\gamma_{2} u_{2}^{2}+\gamma_{1} v_{1}^{2}+\gamma_{2} v_{2}^{2}\right) .
\end{aligned}
$$

In other words, we need to show that

$$
\gamma_{1}\left[\left(\epsilon^{2}-\epsilon\right)\left(u_{1}^{2}+v_{1}^{2}\right)\right]+\gamma_{2}\left[\left(\epsilon^{2}-\epsilon\right)\left(u_{2}^{2}+v_{2}^{2}\right)\right]+2 \epsilon(1-\epsilon)\left(\gamma_{1} u_{1} v_{1}+\gamma_{2} u_{2} v_{2}\right) \leq 0
$$

which is equal to

$$
-\gamma_{1}\left(\sqrt{\epsilon\left(1-\epsilon^{2}\right)} u_{1}-\sqrt{\epsilon\left(1-\epsilon^{2}\right)} v_{1}-\gamma_{2}\left(\sqrt{\epsilon\left(1-\epsilon^{2}\right)} u_{2}-\sqrt{\epsilon\left(1-\epsilon^{2}\right)} v_{2} \leq 0 .\right.\right.
$$

The above inequality holds since $\gamma_{1}, \gamma_{2} \geq 0$.

Hence (30) holds.

Finally, we have to show that,

where $c_{1}>0$ and $\beta>1$.

$$
L(t, \bar{x}, \bar{u}) \leq c_{2}-c_{1}|\bar{u}|^{\beta}
$$

For the case,

$$
L(t, \bar{x}, \bar{u})=x_{1}+x_{2}-\left(\gamma_{1} u_{1}^{2}+\gamma_{2} u_{2}^{2}\right) \leq c_{2}-c_{1}|\bar{u}|^{\beta}
$$

where $c_{2}$ depends on the upper bound on $\left(x_{1}+x_{2}\right)$ and $c_{1}>0$.

Since $\gamma_{1}, \gamma_{2} \geq 0$, we conclude there exists an optimal pair by the existence result from ([10], Theorem 4.1). 
An Ecological Model for Sustainable Forest Management of Eco-system Based on Optimal Control Theory

\subsection{Optimality system}

In previous section we prove the existence of an optimal control pair system for maximizing the functional (27) subject to (24) and (25). In order to derive the necessary conditions on this optimal control pair, we apply Pontryagin's maximum principle [18].

The Lagrangian is defined as follows:

$$
\begin{aligned}
L= & {\left[x_{1}+x_{2}-\left(A_{1} u_{1}^{2}+A_{2} u_{2}^{2}\right)\right]+\lambda_{1} r x_{1}\left(1-\frac{x_{1}}{k}\right)-\beta x_{1}-\gamma x_{1}-\left(1-u_{1}(t)\right) \tau_{1} x_{1}^{3} } \\
& +\lambda_{2} \beta x_{1}-d_{1} E x_{2}-d_{2} x_{2}-\left(1-u_{2}\right) p x_{2}-\left(1-u_{1}\right) \tau_{2} x_{2}^{2}+y_{11}(t)\left(b_{1}-u_{1}\right) \\
& +y_{12}\left(u_{1}-a_{1}\right)+y_{21}(t)\left(b_{2}-u_{2}\right)+y_{22}\left(u_{2}-a_{2}\right)
\end{aligned}
$$

where $y_{11}(t), y_{12}(t), y_{21}(t), y_{22}(t) \geq 0$ are penalty multipliers satisfying,

$$
\begin{aligned}
& y_{11}(t)\left(b_{1}-u_{1}\right)=0, y_{12}\left(u_{1}-a_{1}\right)=0 \text { at } u_{1}^{*}, \\
& y_{21}(t)\left(b_{2}-u_{2}\right)=0, y_{22}\left(u_{2}-a_{2}\right)=0 \text { at } u_{2}^{*} .
\end{aligned}
$$

Theorem 7.3. Given optimal control pair $u_{1}^{*}, u_{2}^{*}$ and the solutions $x_{1}^{*}, x_{2}^{*}$ of corresponding state system (25) and (26), there exists adjoint variables $\lambda_{1}, \lambda_{2}$ satisfying,

$$
\begin{aligned}
& \lambda_{1}^{\prime}=-1-\left[\lambda_{1}\left\{r_{1}\left(1-\frac{2 x_{1}}{k}\right)-\beta+\gamma-3(1-u(t)) \tau_{1} x_{1}^{2}\right]+\lambda_{2} \beta\right. \\
& \lambda_{2}^{\prime}=-1-\left[\lambda _ { 2 } \left\{\left(-d_{1} E-d_{2}-2\left(1-u_{1}(t)\right) \tau_{2} x_{2}-\left(1-u_{2}(t)\right) p\right\}\right.\right.
\end{aligned}
$$

and $\lambda_{1}(T)=\lambda_{2}(T)=0$, the following characterization of transversality conditions hold on the interior of the control set $U$,

$$
\begin{aligned}
u_{1}^{*} & =\min \left\{\max \left(a_{1}, \frac{\lambda_{1} \tau_{1} x_{1}^{3}+\lambda_{2} \tau_{2} x_{2}^{2}}{2 A_{1}}\right), b_{1}\right\}, \\
u_{2}^{*} & =\min \left\{\max \left(a_{2}, \frac{\lambda_{2} p x_{2}}{2 A_{2}}\right), b_{2}\right\} .
\end{aligned}
$$

Proof. (a) These set of controls and corresponding state variables are non-empty.

(b) The set of control $U$ is closed convex set.

(c) On the control set $U$ the objective functional integrand is concave,

(d) The ecological control model (24) and (25) is linear in control variables and is bounded by a linear system in the state and control variables.

Therefore at least one optimal control is existence and the optimal controls are satisfied.

The form of the adjoint and transversality conditions are standard results from Pontryagin's Maximal Principle [5.1], we differentiate the Lagrangian with respect to state $x_{1}$ and $x_{2}$ respectively,

$$
\begin{aligned}
\frac{\partial L}{\partial x_{1}} & =1+\left[\lambda_{1}\left\{r\left(1-\frac{2 x_{1}}{k}\right)-\beta+\gamma-3\left(1-u_{1}(t)\right) \tau_{1} x_{1}^{2}\right]+\lambda_{2} \beta\right. \\
\frac{\partial L}{\partial x_{2}} & =1+\left[\lambda_{2} d_{1} E+d_{2}+p+2 \tau_{2} x_{2}\right] .
\end{aligned}
$$

Thus the adjoint,

$$
\lambda_{1}^{\prime}=-\frac{\partial L}{\partial x_{1}}=-1-\left[\lambda_{1}\left\{r\left(1-\frac{2 x_{1}}{k}\right)-\beta+\gamma-3\left(1-u_{1}(t)\right) \tau_{1} x_{1}^{2}\right]-\lambda_{2} \beta\right.
$$

and

$$
\lambda_{2}^{\prime}=-\frac{\partial L}{\partial x_{2}}=-1-\left[\lambda_{2}\left\{d_{1} E+d_{2}+p+2 \tau_{2} x_{2}\right]\right.
$$

The optimality equations are;

$$
\begin{aligned}
\frac{\partial L}{\partial u_{1}} & =-2 A_{1} u_{1}+\lambda_{1} \tau_{1} x_{1}^{3}+\lambda_{2} \tau_{2} x_{2}^{2}+y_{11}(t)+y_{12}(t) \text { at } t u_{1}^{*} \\
\frac{\partial L}{\partial u_{2}} & =-2 A_{2} u_{2}+\lambda_{2} p-y_{21}+y_{22}(t) \text { at } u_{2}^{*} .
\end{aligned}
$$


Hence we obtain,

$$
\begin{aligned}
u_{1}^{*} & =\frac{\lambda_{1} \tau_{1} x_{1}^{3}+\lambda_{2} \tau_{2} x_{1} x_{2}^{2}-y_{11}(t)+y_{12}(t)}{2 A_{1}}, \\
u_{2}^{*} & =\frac{\lambda_{2} p-y_{21}(t)+y_{22}(t)}{2 A_{2}}
\end{aligned}
$$

In (32) there are three cases for $u_{i}^{*}$ at any time $t^{\prime}$, Case (i):

$$
a_{1}=u_{1}^{*}(t)
$$

Since $a_{1}=u_{1}^{*}(t) \neq b_{1}, y_{11}(t)=0$, then $a_{1}=u_{1}^{*}(t)=\frac{1}{2 A_{1}}\left(\lambda_{1} \tau_{1} x_{1}^{3}+\lambda_{2} \tau_{2} x_{1} x_{2}^{2}+y_{12}(t)\right)$ by $(32)$.

Then, solving for $y_{12}(t)$, gives,

$$
\left.2 a_{1} A_{1}-\lambda_{1} \tau_{1} x_{1}^{3}-\lambda_{2} \tau_{2} x_{1} x_{2}^{2}=y_{12}(t)\right) \geq 0
$$

which implies that,

$$
2 a A_{1} \geq \lambda_{1} \tau_{1} x_{1}^{* 3}(t)+\lambda_{2} \tau_{2} x_{1}^{*}(t) x_{2}^{* 2}(t)
$$

and

$$
a_{1} \geq \frac{1}{2 A_{1}}\left(\lambda_{1} \tau_{1} x_{1}^{* 3}(t)+\lambda_{2} \tau_{2} x_{2}^{* 2}(t)\right)
$$

Case (ii):

$$
a_{1}<u_{1}^{*}(t)<b_{1} .
$$

By the definition of the penalty multipliers, $y_{12} y_{11}=0$, and $(32)$ gives,

$$
u_{1}^{*}=\frac{1}{2 A_{1}}\left(\lambda_{1} \tau_{1} x_{1}^{* 3}(t)+\lambda_{2} \tau_{2} x_{2}^{* 2}\right)
$$

Case (iii):

$$
u_{1}^{*}(t)=b_{1}
$$

Since $u_{1}^{*}(t) \neq a_{1}, y_{12}=0$, and (32) gives,

$$
\left.b_{1}=u_{1}^{*}(t)=\frac{1}{2 A_{1}}\left(\lambda_{1} \tau_{1} x_{1}^{* 3}(t)+\lambda_{2} \tau_{2} x_{2}^{* 2}(t)-y 11(t)\right)\right)
$$

which implies

$$
0 \leq y_{11}=\lambda_{1} \tau_{1} x_{1}^{* 3}+\lambda_{2} \tau_{2} x_{2}^{* 2}(t)-2 A_{1} b_{1}
$$

and

$$
b_{1} \leq \frac{1}{2 A_{1}}\left(\lambda_{1} \gamma_{1} x_{1}^{* 3}+\lambda_{2} \gamma_{2} x_{1} x_{2}^{* 2}(t)\right)
$$

Hence we conclude

$$
u_{1}^{*}= \begin{cases}a_{1} & \text { if } \frac{1}{2 A_{1}}\left(\lambda_{1} \tau_{1} x_{1}^{* 3}+\lambda_{2} \tau_{2} x_{2}^{* 2} \leq a_{1} .\right. \\ \frac{1}{2 A_{1}}\left(\lambda_{1} q_{1} x_{1}^{*}+\lambda_{2} q_{2} x_{2}^{*}\right. & \text { if } a_{1}<\frac{1}{2 A_{1}}\left(\lambda_{1} x_{1}^{* 3}+\lambda_{2} x_{2}^{* 2}\right)<b_{1} . \\ b_{1} & \text { if } \frac{1}{2 A_{1}}\left(\lambda_{1} \tau_{1} x_{1}^{* 3}+\lambda_{2} x_{2}^{* 2}\right) \geq b_{1} .\end{cases}
$$

In compact notation, $u_{1}^{*}(t)=\min \left\{\max \left\{a_{1}, \frac{1}{2 A_{1}} \lambda_{1} \gamma_{1} x_{1}^{* 3}(t)+\lambda_{2} \gamma_{2} x_{1} x_{2}^{* 2}(t)\right\}, b_{1}\right\}$.

In $(33)$ there are three cases for $u_{2}^{*}(t)$ at any time $t$ :

Case (i):

$$
a_{2}=u_{2}^{*}(t) .
$$


We have $y_{21}=0$ and then

$$
a_{2}=u_{2}^{*}(t)=\frac{1}{2 A_{2}}\left(\lambda_{2} p+y_{22}(t)\right)
$$

by $(33)$, then solving for $y_{22}(t)$ gives,

$$
2 A_{2} a_{2}-\lambda_{2} p=y_{22} \geq 0
$$

which implies

$$
2 A_{2} a_{2} \geq-\left(\lambda_{2} p+y_{22}\right.
$$

and

$$
a_{2} \geq \frac{1}{2 A_{2}}\left(\lambda_{2} p\right.
$$

Case (ii):

$$
a_{2}<u_{2}^{*}(t)<b_{2} \text {. }
$$

We have $y_{21}(t)=y_{22}(t)=0$ and $u_{2}^{*}=\frac{1}{2 A_{2}}\left(\lambda_{2} p\right)$

Case (iii):

$$
u_{2}^{*}(t)=b_{2}
$$

since $u_{2}^{*}(t) \neq 0, y_{22}(t)=0$ and (33) gives,

$$
b_{2}=u_{2}^{*}(t)=\frac{1}{2 A_{2}}\left(\lambda_{2} p-y_{21}(t)\right)
$$

which implies

$$
0 \leq y_{21}=\lambda_{2} p-2 A_{2} b_{2}
$$

and

$$
b_{2}^{*} \leq-\frac{1}{2 A_{2}}\left(\lambda_{2} p\right)
$$

Hence we conclude

$$
u_{2}^{*}= \begin{cases}a_{2} & \text { if } \frac{1}{2 A_{2}}\left(\lambda_{2} p \leq a_{2} .\right. \\ \frac{1}{2 A_{2}}\left(\lambda_{2} p\right) & \text { if } a_{2}<\frac{1}{2 A_{2}}\left(\lambda_{2} p\right)<b_{2} . \\ b_{2} & \text { if }-\frac{1}{2 A_{2}}\left(\lambda_{2} p\right) \geq b_{2} .\end{cases}
$$

In compact notation

$$
u_{2}^{*}(t)=\min \left\{\max \left\{a_{2}, \frac{1}{A_{2}}\left(\lambda_{2} p\right), b_{2}\right\} .\right.
$$

The optimality system consists of the state pair with adjoint system with the initial and transversality conditions together with characterization of the optimal control pair,

$$
\begin{aligned}
& u_{1}^{*}(t)=\min \left\{\max \left\{a_{1}, \frac{1}{A_{1}}\left(\lambda_{1} \tau_{1} x_{1}^{* 3}(t)+\lambda_{2} \tau_{2} x_{2}^{* 2}(t)\right)\right\}, b_{1}\right\}, \\
& u_{2}^{*}(t)=\min \left\{\max \left\{a_{2}, \frac{1}{A_{2}}\left(\lambda_{2} p\right), b_{2}\right\} .\right.
\end{aligned}
$$

Note that the initial condition and final time condition in the state system (24) and (25) and co-state system (32) and (33) respectively. 
Journal of Nepal Mathematical Society (JNMS), Vol. 2, Issue 1 (2019); N. Hasan, H. A. Biswas, S. Uddin

\subsection{Uniqueness of the Optimality System}

We can apply the following Lemma for proving the uniqueness of solution of the optimality system for the small time interval.

Lemma 7.1. The function $u^{*}(s)=\min \{\max (s, a), b\}$ is Lipschitz continuous in $s$, where $a<b$ are some fixed positive constraints.

Proof. Consider $s_{1}, s_{2}$ to be real numbers and $a, b$ to be fixed positive constants. We may show the Lipschitz continuity holds in all positive cases for $\max (s, a)$. Similar arguments also hold for $\min \{\max (s, a), b\}$

(i) $s_{1} \geq a, s_{2} \geq a$

$\left|\max \left(s_{1}, a\right)-\max \left(s_{2}-a\right)\right|=\left|s_{1}-s_{2}\right|$

(ii) $s_{1} \geq a, s_{2} \leq a$

$\left|\max \left(s_{1}, a\right)-\max \left(s_{2}, a\right)\right|=\left|s_{1}-a\right| \leq\left|s_{1}-s_{2}\right|$

(iii) $s_{1} \leq a, s_{2} \geq a$

$\left|\max \left(s_{1}, a\right)-\max \left(s_{2}-a\right)\right|=\left|a-s_{2}\right| \leq\left|s_{1}-s_{2}\right|$

(iv) $s_{1} \leq a, s_{2} \leq a$

Hence, $\left|\max \left(s_{1}, a\right)-\max \left(s_{2}-a\right)\right|=|a-a|=0 \leq\left|s_{1}-s_{2}\right|$

and we have Lipschitz continuity of $u^{*}(t)$ in $s$.

Theorem 7.4. If $T$ is sufficiently small and bounded solutions to the optimality system are unique.

Proof. Consider $\left(x_{1}, x_{2}, \lambda_{1}, \lambda_{2}\right)$ and $\left(\overline{x_{1}}, \overline{x_{2}}, \overline{\lambda_{1}}, \overline{\lambda_{2}}\right)$ are two different solutions of the optimality system.

Let $x_{1}=e^{\lambda t} \rho_{1}, x_{2}=e^{\lambda t} \rho_{2}, \lambda_{1}=e^{-\lambda t} \rho_{3}, \lambda_{2}=e^{-\lambda t} \rho_{4}$

and $\overline{x_{1}}=e^{\lambda t} \overline{\rho_{1}}, \overline{x_{2}}=e^{\lambda t} \overline{\rho_{2}}, \overline{\lambda_{1}}=e^{-\lambda t} \overline{\rho_{3}}, \overline{\lambda_{2}}=e^{-\lambda t} \overline{\rho_{4}}$ where $\lambda>0$.

Further, we let

$$
\begin{aligned}
& u_{1}^{*}(t)=\min \left\{\max \left\{a_{1}, \frac{1}{A_{1}}\left(\rho_{3} \gamma_{1} \rho_{1}+\rho_{4} \gamma_{2} \rho_{2}\right)\right\}, b_{1}\right\} \\
& u_{2}^{*}(t)=\min \left\{\max \left\{a_{2}, \frac{1}{A_{2}}\left(\rho_{3} p_{1} \rho_{1}+\rho_{4} p_{2} \rho_{2}\right)\right\}, b_{2}\right\} \\
& \overline{u_{1}^{*}}(t)=\min \left\{\max \left\{a_{1}, \frac{1}{\overline{A_{1}}}\left(\overline{\rho_{3}} \gamma_{1} \overline{\rho_{1}}+\overline{\rho_{4}} \gamma_{2} \overline{\rho_{2}}\right)\right\}, b_{1}\right\} \\
& \overline{u_{2}^{*}}(t)=\min \left\{\max \left\{a_{2}, \frac{1}{A_{2}}\left(\overline{\rho_{3}} p_{1} \overline{\rho_{1}}+\overline{\rho_{4}} p_{2} \overline{\rho_{2}}\right)\right\}, b_{2}\right\} .
\end{aligned}
$$


An Ecological Model for Sustainable Forest Management of Eco-system Based on Optimal Control Theory

Now,

$$
\begin{aligned}
x_{1} & =e^{\lambda t} \rho_{1} \\
\text { or }, \frac{d x_{1}}{d t} & =e^{\lambda t}\left(\rho_{1}^{\prime}+\lambda \rho_{1}\right) \\
\text { or, } \rho_{1}^{\prime}+\lambda \rho_{1} & =e^{-\lambda t} \frac{d x_{1}}{d t} \\
x_{2} & =e^{\lambda t} \rho_{2} \\
\text { or, } \frac{d x_{2}}{d t} & =e^{\lambda t}\left(\rho_{2}^{\prime}+\lambda \rho_{2}\right) \\
\Rightarrow \quad \rho_{2}^{\prime}+\lambda \rho_{2} & =e^{-\lambda t} \frac{d x_{2}}{d t} \\
\lambda_{1} & =e^{-\lambda t} \rho_{3} \\
\text { or }, \frac{d \lambda_{1}}{d t} & =e^{-\lambda t}\left(\rho_{3}^{\prime}-\lambda \rho_{3}\right) \\
\Rightarrow \quad \rho_{3}^{\prime}-\lambda \rho_{3} & =e^{\lambda t} \frac{d \lambda_{1}}{d t} \\
\text { and } d \lambda_{2} & =e^{-\lambda t} \rho_{4} \\
\text { or }, \frac{d \lambda_{2}}{d t} & =e^{-\lambda t}\left(\rho_{4}^{\prime}-\lambda \rho_{4}\right) \\
\rho_{4}^{\prime}-\lambda \rho_{4} & =e^{\lambda t} \frac{d \lambda_{2}}{d t}
\end{aligned}
$$

where,

$$
\begin{aligned}
\frac{d x_{1}}{d t} & =r x_{1}\left(1-\frac{x_{1}}{k}\right)-\beta x_{1}+\gamma x_{1}-\left(1-u_{1}(t)\right) \tau_{1} x_{1}^{3} \\
\frac{d x_{2}}{d t} & =\beta x_{1}-d_{1} E x_{2}-\left(1-u_{2}(t)\right) p x_{2}-\left(1-u_{1}(t)\right) \tau_{2} x_{2}^{2} \\
\frac{d \lambda_{1}}{d t} & =-1-\left[\lambda_{1}\left\{r\left(1-\frac{2 x_{1}}{k}\right)-\beta+\gamma-3\left(1-u_{1}(t)\right) \tau_{1} x_{1}^{2}\right]+\lambda_{2} \beta\right. \\
\frac{\lambda_{2}}{d t} & =-1-\lambda_{2}-d_{1} E-d_{2}-\left(1-u_{2}\right) p-2\left(1-u_{1}\right) \tau_{2} x_{2} \\
u_{1}^{*} & =\min \left\{\max \left(a_{1}, \frac{\lambda_{1} \tau_{1} x_{1}+\lambda_{2} \tau_{2} x_{2}}{A_{1}}\right), b_{1}\right\} \\
u_{2}^{*} & =\min \left\{\max \left(a_{2}, \frac{\lambda_{2} p x_{2}}{A_{2}}\right), b_{2}\right\} .
\end{aligned}
$$

Now subtract the equations for $x_{1}$ and $\overline{x_{1}}, x_{2}$ and $\overline{x_{2}}, \lambda_{1}$ and $\overline{\lambda_{1}}, \lambda_{2}$ and $\overline{\lambda_{2}}$ Using Lemma (7.1) we get,

$$
\left|u_{1}^{*}(t)-\overline{u_{1}}(t)\right| \leq \frac{1}{2 A_{1}}\left|p_{1}\left(\rho_{1} \rho_{3}-\overline{\rho_{1}} \overline{\rho_{3}}\right)+p_{2}\left(\rho_{2} \rho_{4}-\overline{\rho_{2}} \overline{\rho_{4}}\right)\right|
$$

and

$$
\left|u_{2}^{*}(t)-\overline{u_{2}}(t)\right| \leq \frac{1}{2 A_{2}}\left|q_{1}\left(\rho_{1} \rho_{3}-\overline{\rho_{1}} \overline{\rho_{3}}\right)+q_{2}\left(\rho_{2} \rho_{4}-\overline{\rho_{2}} \overline{\rho_{4}}\right)\right| .
$$

Now multiply each equation by an appropriate difference of function and integrate from 0 to $T$. We obtain,

$$
\begin{aligned}
& \frac{1}{2}\left(\rho_{1}-\overline{\rho_{1}}\right)^{2}(T)+\lambda \int_{0}^{T}\left(\rho_{1}-\overline{\rho_{1}}\right)^{2} d t \\
\leq & \int_{0}^{T} r\left|\rho_{1}-\rho_{2}\right|^{2} d t+\beta \int_{0}^{T}|| \rho_{1}-\overline{\rho_{1}}\left|d t+\tau_{1} \int_{0}^{T} e^{\lambda t}\right|\left(1-u_{1}^{*} \rho_{1}\right)-\left(1-\overline{u_{1}^{*}}\right) \rho_{1}|| \rho_{1}-\left.\overline{\rho_{1}}\right|^{2} d t \\
\leq & C_{1} \int_{0}^{T}\left[\left|\rho_{1}-\overline{\rho_{1}}\right|^{2}+\left|\rho_{1}-\overline{\rho_{1}}\right|\right]+C_{2} e^{\lambda T} \int_{0}^{T}\left|\rho_{1}-\overline{\rho_{1}}\right|^{2} d t
\end{aligned}
$$




$$
\begin{aligned}
& \frac{1}{2}\left(\rho_{2}-\overline{\rho_{2}}\right)^{2}(T)+\lambda \int_{0}^{T}\left(\rho_{2}-\overline{\rho_{2}}\right)^{2} d t \\
\leq & \int_{0}^{T} e \beta \int_{0}^{T}\left|\rho_{2}-\overline{\rho_{2}}\right| d t+\tau_{2} \int_{0}^{T} e^{\lambda t}\left|\left(1-u_{1}^{*}\right) \rho_{2}-\left(1-\overline{u_{1}^{*}}\right) \rho_{2}\right|\left|\rho_{2}-\overline{\rho_{2}}\right|^{2} d t \\
& +p \int_{0}^{T} e^{\lambda t}\left|\left(1-\bar{u}_{2}^{*}\right) \rho_{1}-\left(1-\overline{u_{2}^{*}}\right) \overline{\rho_{2}}\right|\left|\rho_{2}-\overline{\rho_{2}}\right|^{1} d t \\
\leq & C_{3} \int_{0}^{T}\left[\left|\rho_{1}-\overline{\rho_{1}}\right|^{2}+\left|\rho_{2}-\overline{\rho_{2}}\right|^{2}\right] d t+C_{4} e^{\lambda T} \int_{0}^{T}\left|\rho_{2}-\overline{\rho_{2}}\right|^{2} d t \\
& \frac{1}{2}\left(\rho_{3}-\overline{\rho_{3}}\right)^{2}(0)+\lambda \int_{0}^{T}\left(\rho_{2}-\overline{\rho_{2}}\right)^{2} d t \\
\leq & \frac{2 \lambda_{1}}{k} \int_{0}^{T}\left|\rho_{3}-\overline{\rho_{3}}\right|^{2} d t-\beta \int_{0}^{T}\left|\rho_{3}-\bar{\rho}_{3}\right| d t+\lambda_{2} \beta \int_{0}^{T} e^{\lambda T}\left|\rho_{3}^{2}-\bar{\rho}_{4}\right| d t \\
\leq & C_{5} \int_{0}^{T}\left[\left|\rho_{2}-\overline{\rho_{2}}\right|^{2}+\left|\rho_{3}-\overline{\rho_{3}}\right|^{2}\right] d t+C_{6} e^{\lambda T} \int_{0}^{T}\left[\left|\rho_{3}-\overline{\rho_{3}}\right|+\left|\rho_{4}-\overline{\rho_{4}}\right|\right] d t \\
& \frac{1}{2}\left(\rho_{4}-\overline{\rho_{4}}\right)(0)+\lambda \int_{0}^{T}\left(\rho_{4}-\overline{\rho_{4}}\right) d t \\
\leq & \lambda_{1} r_{1} w \int_{0}^{T} \rho_{4}^{2}-\overline{\rho_{4}^{2}}\left|d t+\lambda_{2} \beta \int_{0}^{T} e^{\lambda T}\right| \rho_{4}-\overline{\rho_{4}} \mid d t \\
\leq & C_{7} \int_{0}^{T}\left[\left|\rho_{3}-\overline{\rho_{3}}\right|^{2}+\left|\rho_{4}-\overline{\rho_{4}}\right|^{2}\right] d t+C_{8} e^{\lambda T} \int_{0}^{T}\left[\left|\rho_{3}-\overline{\rho_{3}}\right|^{2}+\left|\rho_{4}-\overline{\rho_{4}}\right|^{2}\right] d t .
\end{aligned}
$$

Adding all above four estimates gives,

$$
\begin{aligned}
& \frac{1}{2}\left(\rho_{1}-\overline{\rho_{1}}\right)(T)+\frac{1}{2}\left(\rho_{2}-\overline{\rho_{2}}\right)(T)+\frac{1}{2}\left(\rho_{3}-\overline{\rho_{3}}\right)(0)+\frac{1}{2}\left(\rho_{4}-\overline{\rho_{4}}\right)(0) \\
& +\lambda \int_{0}^{T}\left[\left|\rho_{1}-\overline{\rho_{1}}\right|^{2}+\left|\rho_{2}-\overline{\rho_{2}}\right|^{2}+\left|\rho_{3}-\overline{\rho_{3}}\right|^{2}+\left|\rho_{4}-\overline{\rho_{4}}\right|^{2}\right] d t \\
\leq & \left(\overline{C_{1}}+\overline{C_{2}}\right) e^{3 \lambda t} \int_{0}^{T}\left[\left|\rho_{1}-\overline{\rho_{1}}\right|^{2}+\left|\rho_{2}-\overline{\rho_{2}}\right|^{2}+\left|\rho_{3}-\overline{\rho_{3}}\right|^{2}+\left|\rho_{4}-\overline{\rho_{4}}\right|^{2}\right] d t \leq 0 .
\end{aligned}
$$

where $\bar{C}_{1}, \bar{C}_{2}$ depend on the coefficient and the bounds on $\rho_{1}, \rho_{2}, \rho_{3}, \rho_{4}$. If we consider $\lambda$ and since the natural logarithm is an increasing function such that $\lambda>\bar{C}_{1}+\bar{C}_{2}$ and $T<\frac{1}{3 \lambda} \ln \left(\frac{\lambda-\bar{C}_{1}}{\bar{B}_{2}}\right)$, thus, this gives that

$$
\rho_{1}=\overline{\rho_{1}}, \rho_{2}=\overline{\rho_{2}}, \rho_{3}=\overline{\rho_{3}}, \rho_{4}=\overline{\rho_{4}} .
$$

Hence the solution is unique for small time.

\subsection{Behavior of the Control System}

According to Pontryagin's maximum principle convert the system into a maximizing pointwise Hamiltonian $H$ with respect to $u_{1}, u_{2} \in U$, we get

$$
\begin{aligned}
& H(t, x, u, \lambda)=-\left(A_{1} u_{1}^{2}+A_{2} u_{2}^{2}\right)+\lambda_{1}\left[r x_{1}\left(1-\frac{x_{1}}{k}\right)-e \beta x_{1}+\gamma x_{1}-\left(1-u_{1}\right) \tau_{1} x_{1}^{3}\right] \\
& +\lambda_{2}\left[\beta x_{1}-d_{1} E x_{2}-d_{2} x_{2}-\left(1-u_{2}\right) p x_{2}-\left(1-u_{1}\right) \tau_{2} x_{2}^{2}\right]+\lambda_{3}\left[\left(\alpha_{1}-\frac{\alpha_{2} x_{3}}{a+x_{2}}\right) x_{3}-d_{3} x_{3}\right]
\end{aligned}
$$

where $\lambda_{1}, \lambda_{2}$, and $\lambda_{3}$ be the adjoint variables or co-state variables. Now applying Pontryagin's maximum principle [18] and the existence results for the optimal control [20] we obtain the following proposition: 
An Ecological Model for Sustainable Forest Management of Eco-system Based on Optimal Control Theory

Theorem 7.1. Maximize $J\left(u_{1}, u_{2}\right)$ over $U$ for the optimal control pair $u_{1}^{*}$ and $u_{2}^{*}$ then there exists adjoint variables $\lambda_{1}, \lambda_{2}$ and $\lambda_{3}$ satisfying,

$$
\begin{aligned}
& \frac{d \lambda_{1}}{d t}=-\frac{\partial H}{\partial x_{1}}=-\left[\lambda_{1} r\left(1-\frac{2 x_{1}}{k}\right)-\beta+\gamma-3\left(1-u_{1}\right) \tau_{1} x_{1}^{2}+\lambda_{2} \beta\right] \\
& \frac{d \lambda_{2}}{d t}=-\frac{\partial H}{\partial x_{2}}=\lambda_{2} d_{1} E+d_{2}+\left(1-u_{2}\right) p+2\left(1-u_{1}\right) \tau_{2} x_{2}-\frac{\lambda_{3} \alpha_{2} x_{3}^{2}}{\left(a+x_{2}\right)^{2}} \\
& \frac{d \lambda_{3}}{d t}=-\frac{\partial H}{\partial x_{3}}=-\left[\lambda_{3}\left(\alpha_{1}-\frac{2 \alpha_{2} x_{3}}{a+x_{2}}\right)-d_{3}\right]
\end{aligned}
$$

and with transversality condition as $\lambda_{1}(T)=B_{1}, \quad \lambda_{2}(T)=B_{2}$ and $\lambda_{3}(T)=B_{3}$.

Using optimality condition we have, $\frac{\partial H}{\partial u}=0$ at $u^{*}$ i.e., $\frac{\partial H}{\partial u_{1}}=0$ at $u_{1}^{*}$ and $\frac{\partial H}{\partial u_{2}}=0$ at $u_{2}^{*}$

But

$$
\frac{\partial H}{\partial u_{1}}=-2 A_{1} u_{1}+\lambda_{1} \tau_{1} x_{1}^{3}-\lambda_{2} \tau_{2} x_{2}^{2}=0 \text { at } u_{1}^{*}
$$

Hence,

$$
u_{1}^{*}=\frac{\lambda_{1} \tau_{1} x_{1}^{3}-\lambda_{2} \tau_{2} x_{2}^{2}}{2 A_{1}}, \frac{\partial H}{\partial u_{2}}=-2 A_{2} u_{2}+\lambda_{2} p x_{2}^{2}=0 \text { at } u_{2}^{*} \text { and } u^{*}=\frac{\lambda_{2} p x_{2}}{2 A_{2}} .
$$

On the interior of the control set $U$ the following characterization holds,

$$
\begin{aligned}
& u_{1}^{*}=\min \left\{1, \max \left(0, \frac{\lambda_{1} \tau_{1} x_{1}^{3}-\lambda_{2} \tau_{2} x_{2}^{2}}{2 A_{1}}\right)\right\} \text { and } \\
& u_{2}^{*}=\min \left\{1, \max \left(0, \frac{\lambda_{2} p x_{2}}{2 A_{2}}\right)\right\} .
\end{aligned}
$$

Note that the initial time condition and final condition have in the state system and co-state system respectively.

\subsection{Optimal Harvesting Strategy}

For determining an optimal harvesting strategy we therefore consider the present value $Q=\int_{0}^{\infty} e^{-\delta t} \prod^{(x, u, t) d t}$ where $\Pi$ is economic rent (net revenue) and $\delta$ is the instantaneous rate of annual discount(cf. Arrow and Kurz 1970) at any time $t$. To find the optimal equilibrium using Pontryagin's maximum principle and the associated Hamiltonian function is given by, $\prod(x, u, t)=\left(d_{1} p_{1} x_{2}-c\right) E$.

Thus our objective is to maximize the present value $Q$ subject to the equations (24)-(26) and the control constraints, $u_{\min } \leq u \leq u_{\max }$.

Now the control problem is defined as,

$$
\begin{aligned}
H= & e^{-\delta t}\left[d_{1} p_{1} x_{2}-c\right] E+\lambda_{1}(t)\left[r x_{1}\left(1-\frac{x_{1}}{k}-\beta x_{1}+\gamma x_{1}-\left(1-u_{1}\right) \tau_{1} x_{1}^{3}\right]\right. \\
& +\lambda_{2}(t)\left[\beta x_{1}-d_{1} x_{2} E-d_{2} x_{2}-\left(1-u_{2}\right) p x_{2}-\left(1-u_{1}\right) \tau_{2} x_{2}^{2}\right]+\lambda_{3}(t)\left[\left(\alpha_{1}-\frac{\alpha_{1} x_{3}}{a+x_{2}}\right) x_{3}-d_{3} x_{3}\right]
\end{aligned}
$$

where $\lambda_{1}, \lambda_{2}$ and $\lambda_{3}$ are the adjoint variables.

The singular solution to be optimal if

$$
\sigma(t)=e^{-\delta t}\left(d_{1} p x_{2}-c\right)-\lambda_{2} d_{1} x_{2}
$$

where $\sigma(t)$ known as switching function.

For $E=E_{\max }, \sigma(t)>0$,

$$
\lambda_{2} e^{\delta t}<p_{1}-\frac{c}{d_{1} x_{2}}
$$



and for $E=0 \quad \sigma(t) \geq 0$, we have,

$$
\lambda_{2} \geq e^{-\delta t}\left(p_{1}-\frac{c}{d_{1} x_{2}}\right)
$$

Now for maximum principle, the adjoint variables must satisfy

$$
\frac{d \lambda_{1}}{d t}=-\frac{\partial H}{\partial x_{1}}, \frac{d \lambda_{2}}{d t}=-\frac{\partial H}{\partial x_{2}}, \frac{d \lambda_{3}}{d t}=-\frac{\partial H}{\partial x_{3}}
$$

By considering the equation (38) the adjoint equations and the control equations (24)-(26) become,

$$
\begin{aligned}
\frac{d \lambda_{1}}{d t} & =-\lambda_{1}\left[r\left(1-\frac{2 x_{1}}{k}\right)-\beta+\gamma-3\left(1-u_{1}\right) \tau_{1} x_{1}^{2}\right]-\beta \lambda_{2}, \\
\frac{d \lambda_{1}}{d t} & =\frac{r x_{1} \lambda_{1}}{k}-\beta \lambda_{2}, \\
\frac{d \lambda_{2}}{d t} & =-e^{\delta t} d_{1} p_{1} E+\left(d_{2}+\left(1-u_{2}\right) p+d_{1} E\right) \lambda_{2}-\frac{\alpha_{1} x_{3}^{2}}{a+x_{2}}, \\
\frac{d \lambda_{3}}{d t} & =-\left(\alpha_{1}-\frac{2 \alpha_{1} x_{3}}{a+x_{2}}-d_{3}\right) . \\
\text { Since, } \quad \lambda_{2} & =-e^{-\delta t}\left(p_{1}-\frac{c}{d_{1} x_{2}}\right) .
\end{aligned}
$$

By differentiating, we have,

$$
\frac{d \lambda_{2}}{d t}=\delta e^{-\delta t}\left(p_{1}-\frac{c}{d_{1} x_{2}}\right)
$$

Now from (45) we have,

$$
\begin{aligned}
& \frac{d \lambda_{1}}{d t}-\frac{r x_{1} \lambda_{1}}{k}=\beta e^{-\delta t}\left(p_{1}-\frac{c}{d_{1} x_{2}}\right) \\
& \Rightarrow \lambda_{1}=e^{-\delta t} \frac{b_{1}}{a_{1}+\delta}
\end{aligned}
$$

where

$$
a_{1}=\frac{r x_{1}}{k} \text { and } b_{1}=-\beta\left(p_{1}-\frac{c}{d_{1} x_{2}}\right) .
$$

Using equation (48) and (49), we have

$$
\lambda_{3}=e^{-\delta t} \frac{\left(a+x_{2}\right)^{2}}{\alpha_{1} \alpha_{2} x_{3}^{2}}\left(p_{1} d_{2}-\frac{c d_{2}}{p_{1} x_{2}}-\frac{c E}{x_{2}}-\frac{c \delta}{d_{1} x_{2}}+x_{2} \delta\right) .
$$

Substituting the value of $\lambda_{2}$ in equation (50) we get,

$$
\lambda_{2}=e^{-\delta t} \frac{b_{2}}{a_{2}+\delta}
$$

where

$$
\begin{aligned}
& a_{2}=\left(d_{1} E+d_{2}\right), \\
& b_{2}=p_{1} d_{1} E+\delta p_{1}-\frac{\delta c}{d_{1} x_{2}}+p_{1} d_{2}-\frac{c d_{2}}{p x_{2}}-\frac{c E}{x_{2}} .
\end{aligned}
$$


Now, comparing equation (51) and (43) we have,

$$
p_{1}-\frac{c}{d_{1} x_{2}}=\frac{b_{2}}{a_{2}+\delta} .
$$

Substituting the value of $a_{2}$ and $b_{2}$ we get,

$$
E=\frac{\left(p_{1} d_{1} x_{2}-c\right)\left(d_{2}+\delta\right) x_{2}}{p_{1} d_{1} x_{2}-c-q d_{1}^{2} x_{2}^{2}-c d_{1} x_{2}} .
$$

By using (55) solving the model system (24)-(26), we can get the optimal solution of $x_{2 \delta}$ and $x_{3 \delta}$ and the optimal harvesting effort of forestry biomass $E=E_{\delta}$.

\subsection{Application of Control Strategy}

From different combinations of the controls, two strategies are studied numerically.

(a) Toxic control strategy:

With this strategy, only the control toxicity $u_{1}$ is used to optimize the objective function $J$ by using filtration and reroute of toxic fumes in the water vehicles and industrial zones before making their way to atmosphere. In Fig. (3) and Fig. (4) we have observed that a significant difference between the control of toxicity (with/ without) of the model system.

(b) Combined effect of toxic control and poaching control strategy:

Here, we control on anti-poaching patrol $u_{2}$ to control illegal logging and toxic control $u_{1}$ to control toxicity are used to optimize the objective function $J$. Moreover from the Fig. (3) and Fig. (4) one can observe that the system would lead to direct increase the population size and decrease the industrialization. From Fig. (5) one can predict that if illegal logging is not available then industrialization procedure declines continuously and as $t \rightarrow \infty$ the procedure of the industrialization will extinct.

\section{Numerical Simulations}

For the of demonstrating the analytical results, we have considered some hypothetical data. The parameters of the dynamical model are not based on real world observation because we did not perform a practical case study on a particular species. In order to examine the steady states for interpretation, the model (4)-(6) is analyzed by using the numerical software of Mathematica and the steady state diagrams are obtained using the MATLAB software and numerical value of controls are obtained by using RK 4th order method. Our main objective is to illustrate the final result by numerical simulations considered from a qualitative, rather than a quantitative point of view. Along with the verification of our analytical observations, these numerical results are very much important from the practical point of view.

To verify the model system (4)-(6), we attain the following set of parametric values. $r=1.5, k=100, \beta=0.9, a=2, E=3.8, \gamma=3.5, p=0.1, d_{1}=0.12, d_{2}=0.1, d_{3}=0.785, \alpha_{1}=1.5, \alpha_{2}=$ $3.3, \tau_{1}=0.4, \tau_{2}=0.3, e=0.99$

The coexistence equilibrium point of the system (4)-(6) is $E^{*}(1.63967,1.3794,0.73220)$

From the fig-5.1 we can admit that the model system is stable as all the species are coexist.

After controlling the threaten to verify the control model system (24)-(26), by using RK 4th order method we have found the values of control variables. $u_{1}=0.6090, u_{2}=0.3448$ and final value of the state variables $(2.2509,1.6278,1.6202)$ 
Journal of Nepal Mathematical Society (JNMS), Vol. 2, Issue 1 (2019); N. Hasan, H. A. Biswas, S. Uddin

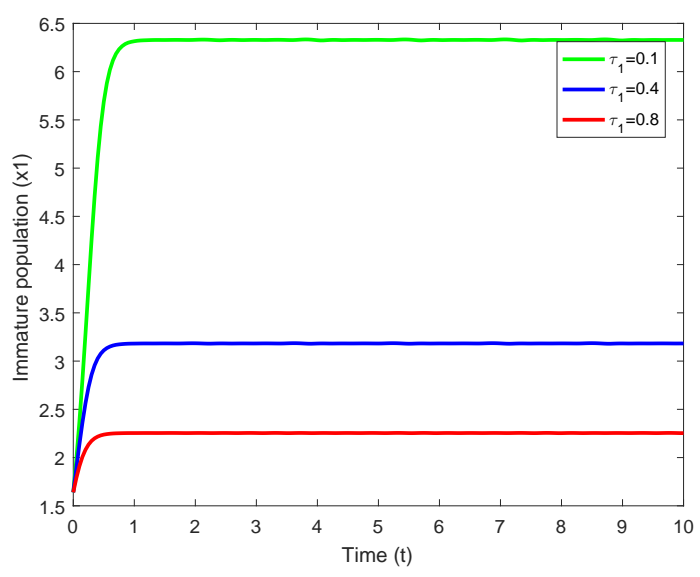

Figure 3: Immature Population (Juvenile trees)

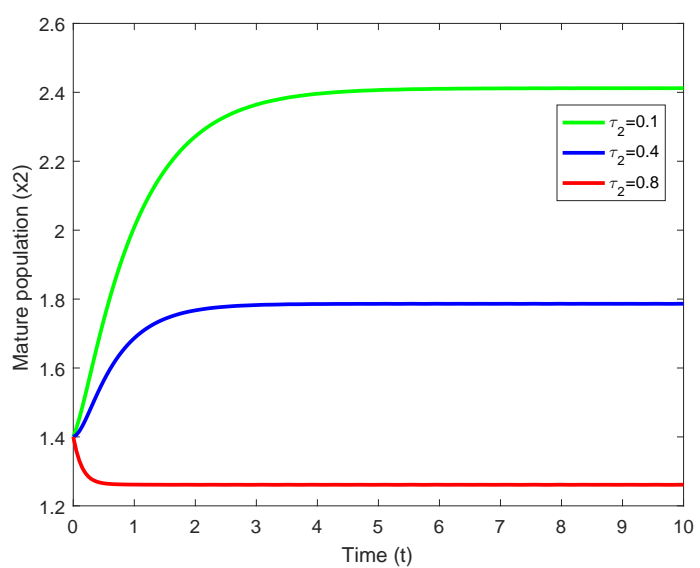

Figure 4: Mature Population (Adult trees)

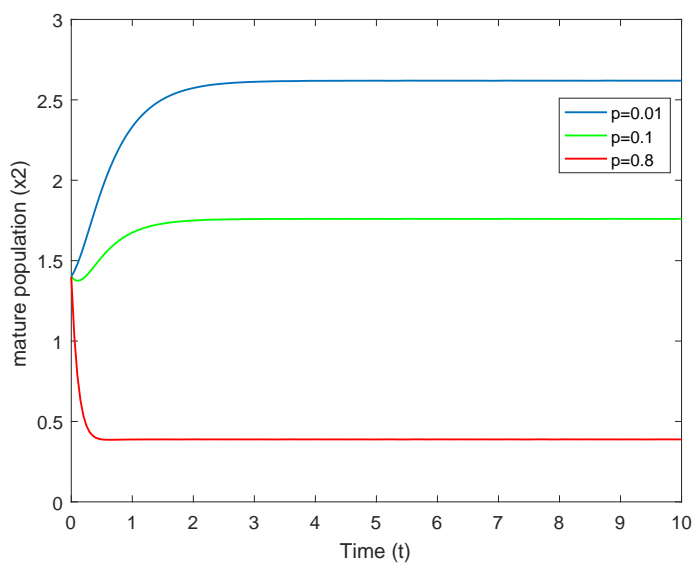

Figure 5: Mature Population (Adult trees) 


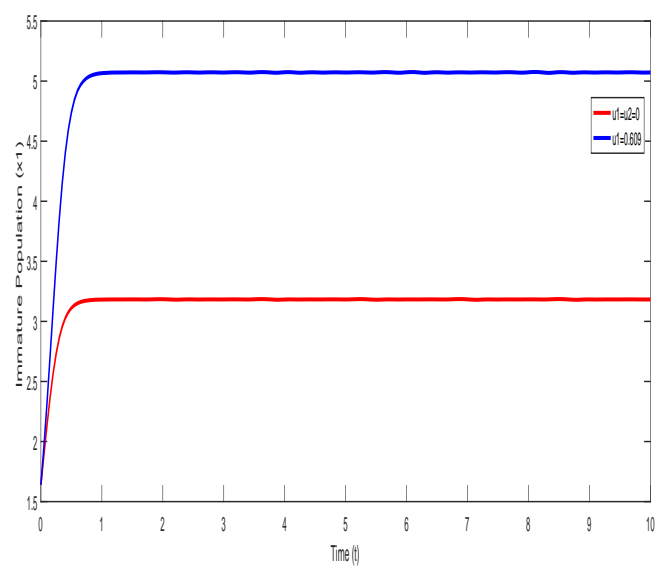

Figure 6: Immature Population (Juvenile trees)

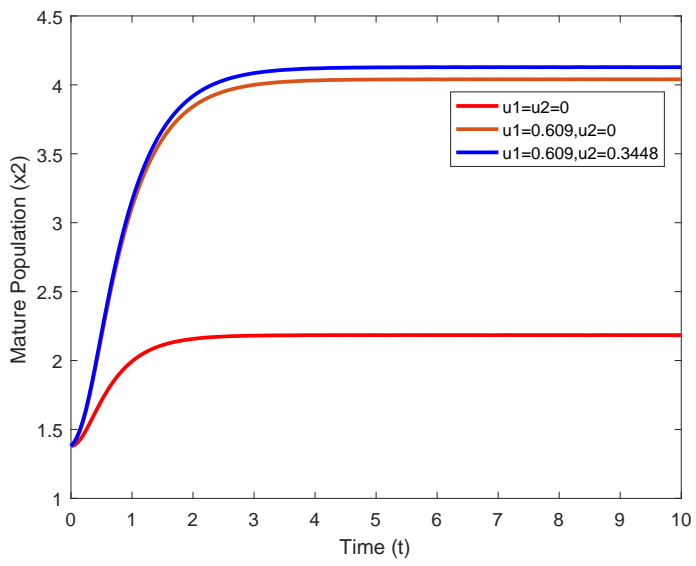

Figure 7: Mature Population (Adult trees)

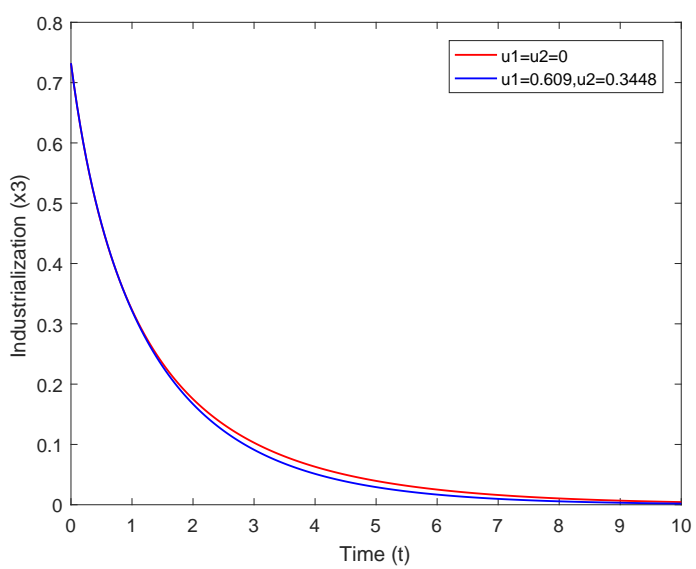

Figure 8: Number of Industries 
Journal of Nepal Mathematical Society (JNMS), Vol. 2, Issue 1 (2019); N. Hasan, H. A. Biswas, S. Uddin

\section{Conclusions}

In this paper, we have presented a threatened age structured forestry based biomass model together with industrialization as a state variable using a deterministic system of differential equations. The threats are poaching and external toxicity. Leslie-Gower and modified Leslie-Gower functional responses are used for the problems discussed in this study. The local and global stability conditions are obtained (parametric). Controls are introduced to the system which are anti-poaching patrols for controlling poaching, filtration and re-route of toxic fumes in the water vehicles and industries for controlling toxicity. To investigate the effect of optimal control, we use one control at a time, the combination of two controls is used at a time while setting other(s) to zero to compare the effects of the control strategies on the eradication of threats to the system. Additionally, the case of all controls has also been taken into consideration. Our numerical simulations suggest that the use of two controls has highest impact on the control of the system threats. We have also shown through graphs (Figs. 3,4) that the immature and mature population has decreased when the toxicity has increased and in Fig 5 we have observed the effect of poaching the mature tree has decreased gradually. In Figs. 6,7 and 8 we have observed that if controls are applied then the immature forestry biomass, mature trees increase significantly and industrialization declines. To find the optimum equilibrium level Pontryagin's maximum principle has been applied. In future, One can able to extend the proposed model system through various ecological implications to understand better the real world ecological system.

\section{Acknowledgments}

The first author gratefully acknowledges the financial support provided by the University Grant Commission, Bangladesh (UGC/1,157/ M.Phil and PhD/2016/5343, Date: 22/06/2016.

\section{References}

[1] Aziz-Alaoui MA (2002) Study of a Leslie-Gower-type tritrophic population model. Chaos Solitons 6 Fractals., 14(8): 1275-1293.

[2] Aziz-Alaoui MA, Okiye MD (2003) Boundedness and global stability for a predator-prey model with modified Leslie-Gower and holling-type II scemes. Appl. Math. Lett., 16(7): 1069-1075.

[3] Chaudhary M, Dhar J Sahu GPV (2013) Mathematical model of depletion of forestry resource: effect of synthetic based industries. Int. J Biol. Vet. Agric Food Eng.,7(4): 130-134.

[4] Chaudhary M, Dhar J, Sahu GPV (2013) Forest biomass conservation with synthetic industry: a mathematical model. In: Engineering (NUiCONE), Nirma University international conference on IEEE: $1-5$.

[5] Chaudhuri K (1988) Dynamic optimization of combined harvesting of a two species fishery. Ecol. Model, 41(1): 17-25.

[6] Chen F (2005) On a nonlinear non autonomous predator-prey model with diffusion and distributed delay. Appl. Math. Lett., 180(1): 33-49.

[7] Chen LJ, Chen F (2009) Global stability of a Leslie-Gower Predator-prey model with feedback controls. Appl. Math. Lett., 29(9): 1330-1334.

[8] Dhar J, Singh H (2004) Modeling the depletion of forestry resource by wholly dependent industrialization in two adjoining habitat. Kobe J. Math., 21: 1-13.

[9] Dubey B, Sharma S, Sinha P, Shukla J (2009) Modeling the depletion of forestry resources by population and population pressure augmented industrialization. Appl. Math. Model, 33(7): 30023014 .

[10] Dubey B, Patra A, Sahani S (2014) Modeling the dynamics of a renewable resource under harvesting with taxation as a control variable. Appl. Math., 9(2): 592-621.

[11] Garecia O (2013) Forest stands as dynamical systems: an introduction. Modern Appl. Sci., 7(5): 32.

[12] Ghosh B, Kar T (2014) Sustainable use of prey species in prey-predator system: jointly determined ecological thresholds and economics trade offs. Ecol. Model, 272: 49-58. 
An Ecological Model for Sustainable Forest Management of Eco-system Based on Optimal Control Theory

[13] Gupta R, Chandra P (2013) Bifurcation analysis of modified Leslie-Gower predator-prey model with michaelis-menten type prey harvesting. J.Math.Anal. Appl., 398(1): 278-295.

[14] Leslie P (1958) A stochastic model for studying the properties of certain biological systems by numerical methods. Biometrika: 16-31.

[15] Misra A, Lata K, Shukla J(2014) A mathematical model for the depletion of forestry resources due to population presure augmented industrialization. Int. J Model Simul sci. Comput, 5(1): 130022.

[16] Misra A, Lata K (2015) A Depletion and conservation of forestry resources: a mathematical model. Differt. Equ. Dyn. Syst., 23(1): 25-41.

[17] Zhang N, Chen F, Su Q, Wu T (2011) Dynamics behaviors of a harvesing Leslie-Gower predator-prey model. Dis. Dyn. Nat. Soc., 18.

[18] Chaudhary M, Dhar J, Misra P (2015) A mathematical model for the conservation of forestry biomass with an alternative resource for industrialization: a modified Leslie Gower interaction. Model. Earth Syst. Environ. 1: 43, 1: 43.

[19] Pontryagin L. S, Boltyanskii V. G, Gamkrelize R. V, Mishchenko E. F (1967) The Mathematical Theory of Optimal Processes. Wiley, New York.

[20] Lukes D. L (1982) Differential Equations: Classical to Controlled. Academic Press, New York.

[21] Lenhart S, Workman J (2007) Optimal Control Applied to Biological Models. Boca Raton, Chapmal Hall, CRC.

[22] Fleming W, Rishel R (2005) Deterministic and Stochastic Optimal Control. Springer-Verlag, New York.

[23] UFAO (2010) Global forest resources assessment. UN Food and Agriculture Organization, Rome.

[24] Khaine I, Woo SY (2015) An overview of interrelationship between climate change and forest. For Sci. Tech., 11 (1): 11-18.

[25] Clark CW (1976) Mathematical Bio-economics: The mathematics of conservation. Wiley, Hoboken. 